\title{
Journal of Hepatology
}

\section{IMPROVED MANAGEMENT OF ADVERSE EVENTS WITH TAILORED SORAFENIB DOSING PROLONGS SURVIVAL OF HEPATOCELLULAR CARCINOMA PATIENTS

\author{
--Manuscript Draft--
}

\begin{tabular}{|c|c|}
\hline Manuscript Number: & JHEPAT-D-19-00748R1 \\
\hline Article Type: & Original Article \\
\hline Section/Category: & Hepatic and Biliary Cancer \\
\hline Keywords: & $\begin{array}{l}\text { hepatocellular carcinoma; sorafenib; adverse events; outcome; prognosis; learning } \\
\text { curve. }\end{array}$ \\
\hline Corresponding Author: & $\begin{array}{l}\text { Francesco Tovoli } \\
\text { University of Bologna } \\
\text { Bologna, ITALY }\end{array}$ \\
\hline First Author: & Francesco Tovoli \\
\hline \multirow[t]{10}{*}{ Order of Authors: } & Francesco Tovoli \\
\hline & Luca lelasi \\
\hline & Andrea Casadei Gardini \\
\hline & Alessandro Granito \\
\hline & Francesco Giuseppe Foschi \\
\hline & Giulia Rovesti \\
\hline & Giulia Negrini \\
\hline & Giulia Orsi \\
\hline & Matteo Renzulli \\
\hline & Fabio Piscaglia \\
\hline Abstract: & 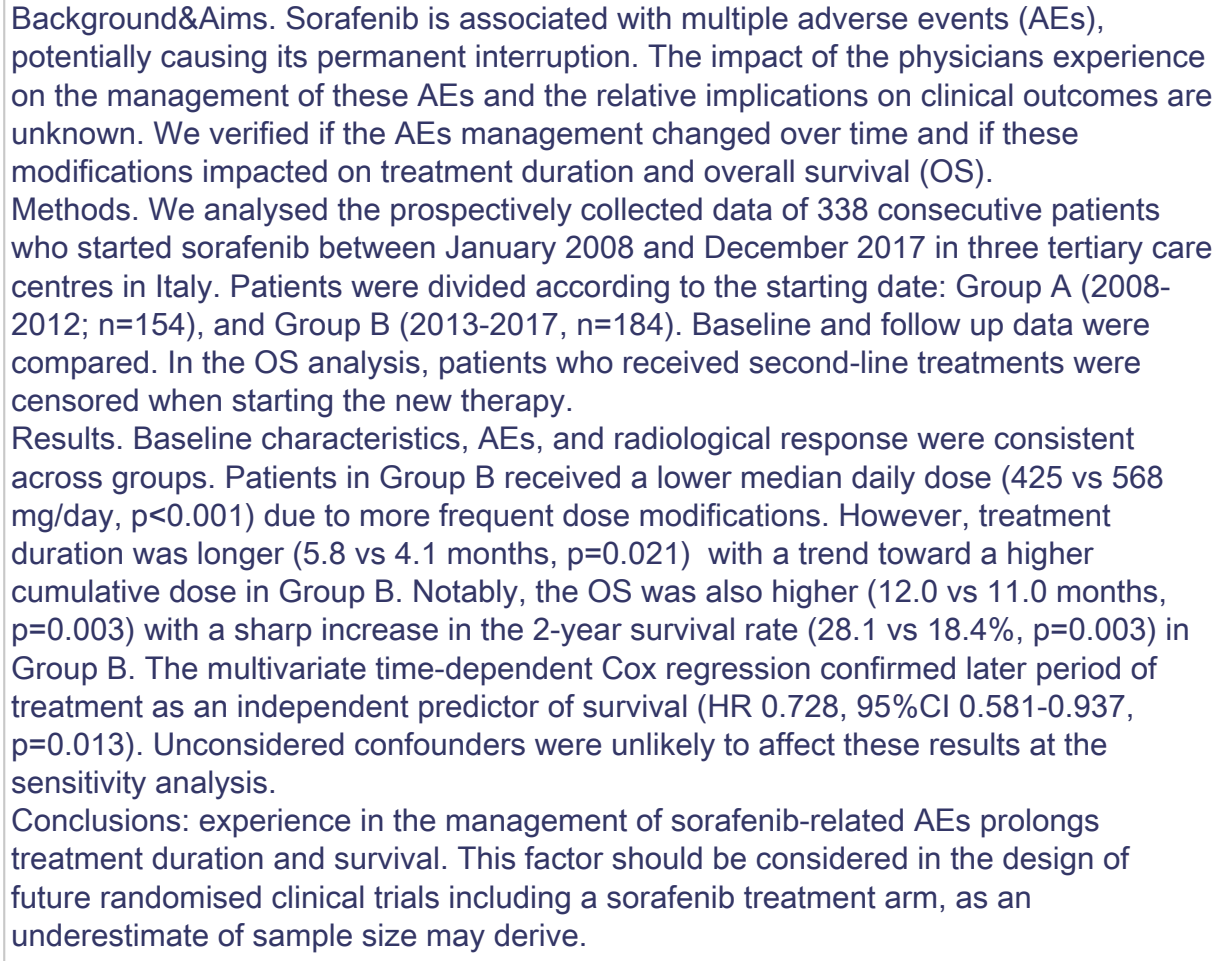 \\
\hline
\end{tabular}

Response to Reviewers: 


\section{DEPARTMENT OF MEDICAL AND SURGICAL SCIENCES University of Bologna, Italy}

July 10, 2019

To the Editor of Journal of Hepatology

Dear Professor,

Please find enclosed the revised version of our manuscript titled "IMPROVED MANAGEMENT OF ADVERSE EVENTS WITH TAILORED SORAFENIB DOSING PROLONGS SURVIVAL OF HEPATOCELLULAR CARCINOMA PATIENTS" to be submitted as an Original Article for consideration of publication.

First of all, we would like to thank both the Editor and the Reviewers for their interest in the original version of our paper and for their precious suggestions. We included a detailed point-to-point response to all of the considerations raised by the Editor and Reviewers. We feel that these suggestions helped us into improving the quality of our paper. However, we are open to any further option which could be helpful in clarifying any remaining doubt and in further improving our manuscript.

On behalf of the Authors

Francesco Tovoli, MD 


\section{Journal of Hepatology}

\section{Revised Submission Checklist}

This form must be completed and submitted for all revised manuscripts. Without this form the manuscript will be returned to the corresponding author for completion.

\begin{tabular}{l|l|}
\cline { 2 - 2 } Corresponding Author: & Francesco Tovoli \\
\cline { 2 - 2 } Manuscript Number: & JHEPAT-D-19-00748
\end{tabular}

Below, provide the page number(s) or figure legend(s) where the information can be located. Please make sure that all the information requested below is present in the manuscript.

\section{1) Submission}

a) Title page: COI, Financial support, Authors' contributions, keywords.

b) Structured abstract and lay summary

c) All tables and figures included, numbered correctly, with legends ( $p$ value and statistical test)

d) Supplementary data included in a single, separate word file

e) A detailed point by point response to reviewers comments and changes highlighted in text

f) All authors to complete and upload an ICMJE conflict of interest form.

g) Graphical abstract

\section{2) Materials and methods}

a) Completed the CTAT form for all reagents and resource to be added to supplementary material

b) Identify the source and authentication of cell lines

c) Identify animal species, number of animals used, strain, sex and age.

d) For animal studies include a statement of compliance with ethical regulations and identify the committee(s) approving the experiments.

e) For qPCR data provide information according to the Minimum Information for Publication of Quantitative Real-Time PCR Experiments (MIQE) guidelines

\section{3) Human subjects}

a) Identify the committee(s) approving the study protocol.

Page 6

b) Include a statement confirming that informed consent was obtained from all subjects.

\begin{tabular}{|c|}
\hline \\
\hline \\
\hline \\
\hline \hline \\
\hline$Y$ \\
\hline$Y$ \\
\hline$Y$ \\
\hline$Y$ \\
\hline$Y$ \\
\hline
\end{tabular}

Completed, or reported on page(s) or figure legend(s):

\section{Page 6}

Not applicable

Not applicable

Not applicable

Not applicable

Page 6

Not applicable 


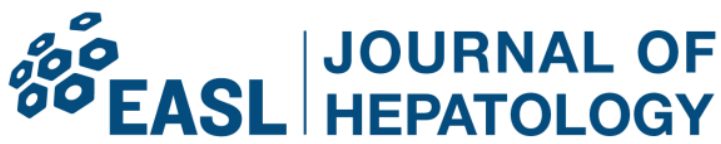

c) For randomized studies report the clinical trial registration number (at ClinicalTrials.gov or equivalent).

d) For phase II and III randomized controlled trials:

a. Please refer to the CONSORT statement and submit the CONSORT checklist with your submission.

Not applicable

b. Include the study protocol and statistical plan

Not applicable

e) Identify the inclusion/exclusion criteria in the selection process for the patients included in the study

Page 3

4) Statistics

a) State what statistical tests were completed and why

Page 6

b) Explain the sample size and how this size provides an adequate power to detect a pre-specified effect size.

Not applicable

5) Data deposition (Provide accession codes for deposited data)

a) When using public databases:

a. Identify the source and include a valid link

Not applicable

b. When using databases that require permission, include a statement confirming that permission was obtained

Not applicable

b) Data deposition in a public repository is mandatory for:
a. Protein, DNA and RNA sequences
b. Microarray data

Deposition is strongly recommended for many other datasets for which structured public repositories exist

Not applicable

Not applicable

Not applicable 


\section{JOURNAL \\ OF HEPATOLOGY}

\section{Journal of Hepatology \\ CTAT methods}

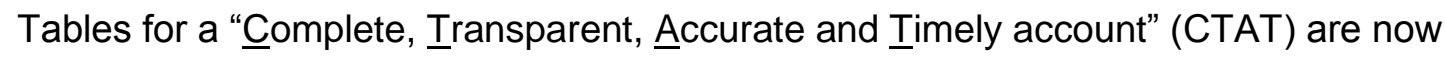
mandatory for all revised submissions. The aim is to enhance the reproducibility of methods.

- Only include the parts relevant to your study

- Refer to the CTAT in the main text as 'Supplementary CTAT Table'

- Do not add subheadings

- Add as many rows as needed to include all information

- Only include one item per row

If the CTAT form is not relevant to your study, please outline the reasons why:

\subsection{Antibodies}

\begin{tabular}{|l|l|l|l|l|}
\hline Name & Citation & Supplier & Cat no. & Clone no. \\
\hline & & & & \\
\hline
\end{tabular}

\section{$1.2 \quad$ Cell lines}

\begin{tabular}{|l|l|l|l|l|l|}
\hline Name & Citation & Supplier & Cat no. & Passage no. & $\begin{array}{l}\text { Authentication } \\
\text { test method }\end{array}$ \\
\hline & & & & & \\
\hline
\end{tabular}

\subsection{Organisms}

\begin{tabular}{|l|l|l|l|l|l|l|}
\hline Name & Citation & Supplier & Strain & Sex & Age & $\begin{array}{l}\text { Overall } \mathbf{n} \\
\text { number }\end{array}$ \\
\hline & & & & & & \\
\hline
\end{tabular}

\subsection{Sequence based reagents}

\begin{tabular}{|l|l|l|}
\hline Name & Sequence & Supplier \\
\hline & & \\
\hline
\end{tabular}

\subsection{Biological samples}

\begin{tabular}{|l|l|l|}
\hline Description & Source & Identifier \\
\hline & & \\
\hline
\end{tabular}

\subsection{Deposited data}

\begin{tabular}{|l|l|l|}
\hline Name of repository & Identifier & Link \\
\hline
\end{tabular}




\section{JOURNAL \\ OF HEPATOLOGY}

\subsection{Software}

\begin{tabular}{|l|l|l|}
\hline Software name & Manufacturer & Version \\
\hline SPSS & SPSS Inc & 23.0 \\
\hline
\end{tabular}

1.8 Other (e.g. drugs, proteins, vectors etc.)

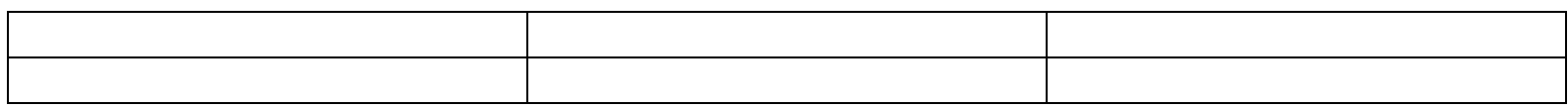

1.9 Please provide the details of the corresponding methods author for the manuscript:

\section{Francesco Tovoli, MD}

Department of Medical and Surgical Sciences, University of Bologna

Via Massarenti 9, 40136 Bologna, Italy

Phone number : +390512142214, Fax number +390516362725

E-mail: francesco.tovoli2@unibo.it

2.0 Please confirm for randomised controlled trials all versions of the clinical protocol are included in the submission. These will be published online as supplementary information. 


\section{Point-to-point response}

Editors' comments: Your manuscript has been evaluated by two experts in the field and by the board of editors. Although the reviewers agree that the manuscript is interesting and has potential future therapeutic implications, they raised substantive concerns. Moreover, the authors have to mention a very similar paper has been published recently (DOI: 10.1159/000497161). Under these circumstances the journal is unable to accept your manuscript in its present form, but the journal will accept to review a revised version of your manuscript if you feel you can deal with all the reviewers' comments.

We would like to thank the Editor for the interest in our paper. The paper DOI: $10.1159 / 000497161$ is indeed similar to our work. The main differences are represented by the multicenter design, the larger dimension of the two cohorts (which allowed more elaborate analyses of the prognosticators), the censoring of patients at the beginning of the second line treatment, and the confirmation of the actual effect of the period of treatment in a multivariate analysis. The latter point was lacking in the previous paper and, in our opinion, represent an important element of novelty. The paper by Raoul et al had been previously mentioned only in the Discussion. We further quoted this paper in the Introduction. This paper is now being referenced in the following sentences

INTRODUCTION: "A very recent monocentric study [10] suggested that the experience in managing sorafenib AEs has improved overtime, but rigorous multicentric studies taking into account also time-dependent variables, addressing the possible confounding factor of second-line treatments, and providing a confirmation of the survival benefit through multivariable regressions models are still lacking"

DISCUSSION: "Only very recently, Raoul and colleagues [10] reported similar results in a retrospective monocenter study. Their report of an increase in treatment duration from 4.3 months in 2008-2012 vs 5.9 months in 2013-2017 is strikingly similar to our results. Our study provides a validation of these initial results through a large multicenter collaboration. DISCUSSION:" In the aforementioned study by Raoul et al. [10], the OS was in fact significantly higher in the last five years (12 vs 8 months) in the univariate analysis, but it was not possible to confirm this result also in a multivariate regression."

\section{Further notes to the Editor}

1. We updated the $\mathrm{COI}$ of the Authors as of $10 \mathrm{Jul} 2019$

2. We added a lay summary as per Revised Manuscript Checklist point $1 b$.

3. We added a specification about the identity of Ethic Commettees approving the study as per Revised Manuscript Checklist point $3 a$.

4. We added a statement about the statistical software used for statistical analyses as per CTAT table point 1.7

5. Discussion: we added reference 29 to provide a very updated guide to the rapidly evolving scenario of first line trials using sorafenib as a comparator drug.

Reviewer \#1: This study represents a retrospective analysis of prospectively collected data on patients receiving sorafenib for advanced hepatocellular carcinoma. The topic is clinically relevant, carefully conducted and the results are interesting, despite the inherent risk of bias due to the retrospective design of the study and possible time/era related factors. The message in the discusion is clear and the conclusions are of interest to the field.

We thank the reviewer for appreciating our paper.

Reviewer \#2: I read with great interest the paper entitled "Improved management of adverse events with tailored sorafenib dosing prolongs survival of hepatocellular carcinoma patients". In this manuscript, the authors try to expose the impact of the acquired experience with sorafenib in the outcomes of patients with hepatocellular carcinoma treated with this drug. For this aim, they designed a multicenter retrospective study where three centers were invited to participate. These authors show that the better understanding of safety profile and its management allow a longer exposure to the drug, what leads to a longer survival.

Despite the interest of the work, it lacks novelty, as the authors expose in the manuscript in regards of the work by Raoul et al. Nevertheless, the description of events and statistical approaches that were done for this manuscript are very valuable. 


\section{METHODS}

1) Why did you consider mRECIST criteria instead of RECIST criteria? Modified RECIST is not validated for the response assessment as may overestimate objective response.

We agree that RECIST 1.1 are more broadly accepted than mRECIST in the evaluation of the response to systemic treatments. Therefore, we modified our manuscript adopting RECIST instead of mRECIST. After this re-categorization, only 2 of the 4 complete responses and less than a half of the partial responses were confirmed. Instead, both criteria equally identified progressors, so the rate of PD was identical. The difference in terms of response between the study groups remained nonsignificant $(p=0.688)$

We modified the Methods - Biochemistry and imaging evaluation section as follows: "Radiologic evaluation of response during follow-up was done by CT-scan according to the Response Evaluation Criteria In Solid Tumours (RECIST) v1.1 [15]. These criteria were preferred over the modified RECIST (MRECIST) [16,17] due to the possible overestimation of tumor necrosis in the setting of systemic treatments by the latter criteria [18]." We also updated Figure 1 with the new results.

2) In terms of sorafenib discontinuation, radiological progression was enough for discontinuing the drug in those patients elegible for secondline drugs. In the first period of the study there were no approved drugs, and second-line trials were scarce. Did you evaluate this in your analyses? This may impact in the evaluation of outcomes (For instance, those patients in the first period could have been treated for longer periods as sorafenib could not have been discontinued because of the lack of second line options).

We were aware of this possible bias when the study was designed. Nonetheless, To reduce the risk of bias we decided to censor the patients at the start of the second-line treatment. This choice was necessary to avoid he confounding factor of second line therapies on survival, but led to a greater risk of losing follow-up information in the second period of the study. Therefore we evaluated the C-index to verify the entity of this loss. This test confirmed a better completeness in the first period due to the paucity of second-line trials in that timeframe, however, the loss of completeness in the second group was negligible in absolute and clinical terms, even if there are no tests to perform an analytical comparison of the two C-index values (please also see response to Point 5). Indeed, even if the risk of a significant bias was ruled out, we agree with the comment by the reviewer about the possibility of a more prolonged sorafenib treatment in the first period (when less trials were available). This bias, however, would have been significant only in the instance of an actually longer period of treatment and OS in the first group. As such, the impact of our findings is not lessened but rather strengthened by this factor. We thank the reviewer for this useful insight and we added the following lines to the Discussion session:

"This longer treatment duration in the second period is even more striking if we take into the account the possible interference derived by the absence of approved drugs and the relative paucity of clinical trials in the first period of the study. This different clinical setting, in fact, could have lead to longer treatment durations in the first period, as sorafenib could not have been discontinued to offer second line options, as they were lacking at that time"

3) Why did you consider 12 and 24 months as cut-offs for landmark analyses? What is the rational for it in the setting of advanced HCC?

We agree that more information about this choice are needed. The 12 months cut-off was chosen to reflect the most recently described median overall survival of sorafenib-treated patients (REFLECT trial). The reasons for the 24 months cut off was twofold: first, it describes the exact double value of the median survival; second, it was recently adopted as a cut-off to define long-survivors. The same cut-off were used in the landmark analyses of an ancillary study of the REFLECT trial (Kudo et al, ABS 186, ASCO GI 2019 San Francisco)

We added the following lines to the Methods section: The choice of these cut-offs derived from the most recently described median OS of sorafenibtreated patients in the REFLECT trial [9] and by the recently proposed cut off to define long survivors to sorafenib [7,21], respectively. The same cut-off were used in the landmark analyses of a recent ancillary study of the REFLECT trial [22].

\section{RESULTS}

4) Study population: You have not explained the rational for choosing the two periods. Therefore, what is the rational for $2008-2012$ and 2013-2017, instead of other periods?

We are sorry for having omitted this critical information. We added the following lines in the Design of the study section: "For the purpose of this study, the whole 2008-2017 timeframe was divided in two exactly equally long periods (January 2008 - December 2012 and January 2013 December 2017).

5) Baseline characteristics: Did you evaluate the possibility of DAA treatment for HCV-related HCC? This may impact in the differences of the treatment duration between the two periods, as patients achieving SVR are at lower risk of liver decompensation, and therefore, may be treated 
for longer periods of time. Moreover, if you have patients treated with DAA, it would be interesting to know if they have a more aggressive tumor behavior or not.

We considered the possibility of a greater number of HCV patients being in SVR in the second period due to a larger availability of DAA. However, this possibility was not confirmed when we compared the rate of SVR in HCV patients in the two periods (12.4 and $14.1 \%$ in the first and second period, respectively). The risk of liver decompensation due to an active HCV replication was consequently similar in the two groups. These results are probably due to the regulatory policies of the Italian Drug Agency, which do not allow the prescription of DAA in patients with active HCC, regardless of its stage (as a consequence, viraemic patients with early- or intermediate-stage HCV-related HCC who transitioned to the advanced stage never received DAAs). On the contrary, patients could have received DAA only if they never had HCC or if they had a complete response to previous treatments. Both of these hypothesis accounted only for a minority of our patients. However, we agree with the reviewer about the opportunity of adding additional information about HCV-positive patients. Therefore we modified the part of the "Baseline characteristics" describing viral infections as follows:

"Amongst patients with HCV-related liver disease, the rate of HCV-RNA positive patients was 87.6 and $85.9 \%$ in Groups A and B, respectively $(p=0.748)$. These proportions accounted for 19 and 26 patients in sustained virological response in the first and second period, respectively. All of the 19 patients in the 2008-2012 period had achieved a sustained virological response after interferon-based treatments. Amongst the 26 patients who started sorafenib in the 2013-2017 period, 18 had eradicated the HCV infection with interferon-based treatments and 8 with second-generation direct antiviral agents."

We also would like to thank the reviewer for suggesting to verify whether post-DAA HCC had a more aggressive tumor behavior. We added the following lines:

METHODS: "Patients with HCV-related cirrhosis in sustained virological response after a viral eradication with direct anti-viral agents were considered a group of special interest, as recent reports suggested that they might develop a more aggressive form of HCC"

RESULTS: "With the limitations of the reduced sample size $(n=8)$, patients with post-DAA HCC had a more frequent onset directly in the advanced stage (75.0\%), but their response to sorafenib was not worse compared to the remaining study population (disease control rate $62.5 \%$ ). Also, the OS of these patients was not significantly impaired (median 12.0 months)."

DISCUSSION: "The eight patients previously treated with direct antiviral agents had a more frequent onset in the advanced stage as previously described by Reig at al, but their response to sorafenib was not different from that of the remaining study population."

6) Follow up: It looks like the C-index is different among the two groups. Did you evaluate if differences were statistically significant? And if so, what is your explanation for it?

To the best of our knowledge, the C-index as designed by Clark et al is meant to provide a descriptive analysis of the completeness of the followup but does not allow an analytical comparison between groups. That said, we agree that there is a slight difference between the two groups that is justified by a greater number of patients being censored due to the availability of post-sorafenib treatments. We reported these considerations into the paper:

DISCUSSION: "Censoring the survival of patients who received second-line treatments excluded the longer OS was justified by improvements in oncological treatments. This choice caused a slightly lower completeness of the follow up in the second group, which, however, remained very high in absolute terms".

7) Adverse events evaluation and management: Did you check if there were differences in terms of patients' comorbidities? This may be an important issue, as we are probably more conservative treating some patients with some clinical conditions (heart diseases, etc.) that what we were before. This may also impact in the length of treatment in the periods analyzed.

Comorbidies are indeed a significant problem in the prescription and management of sorafenib, especially cardiovascular diseases. We actually found a relatively low proportion of patients with cardiovascular comorbidities, with no significant differences across the periods of treatment. This finding is probably justified by our basically strict adherence to the indications and contraindications of sorafenib since its licensing in 2008, especially in terms of cardiovascular comorbidities. In detail, sorafenib was prescribed only in very selected cases of patients with symptomatic congestive heart failure or coronary hearth disease, based on the earlier evidence of potentially severe adverse events of sorafenib and other TKIs in these cases (PMID: 18838713)(PMID: 19734999). We reported the following information:

METHODS - Baseline evaluation: "We recorded the following data for each patient at the time of the first prescription of sorafenib: time elapsed since the first diagnosis of HCC, previous treatments for HCC, aetiology of the underlying liver disease, presence/absence of liver cirrhosis, and comorbidities." 
DISCUSSION - "First, the baseline characteristics of the patients were similar in the two groups in terms of tumour burden, liver function, performance status, comorbidities, and number of previous TACE procedures, making the hypothesis of an improved referral of the patients unlikely as a possible alternative explanation".

8) From 2008 to 2017, the classification of adverse events has changed (CTCAE 3.0, CTCAE 4.03). Did you check if the classification of severity was comparable among the two groups? For instance, definition of grade $2 / 3$ of rash, dry skin, hypertension, etc. has changed between these two versions. This concern is applicable also to table 2 .

The reviewer raises an important point. The classification of severity of adverse events had been made comparable for the two groups In the original version of the paper as we were aware of this potential bias. This operation was possible as clinical records included not only the grade of each adverse event but also a brief description. For instance, we were able to re-codify hypertension as blood pressure values and prescribed anti-hypertensive drugs were recorded in the clinical data, instead of a mere report of "hypertension G2". Similarly, in case of skin rash, we recorded the percentage of the body surface area, the characteristics of the rash, the presence of symptoms (such as pruritus), etc. We added the following information:

METHODS - Adverse events evaluation and management: "The medical records included both a brief description of each AE as well as its coding according to the Common Terminology Criteria for Adverse Events (CTCAE) used at the time of registration. Since the CTCAE version was upgraded from version 3.0 to version 4.03 in June 2010, all of the AEs occurred before that date were re-codified according to the newer version to allow a correct comparison between groups."

9) Imaging evaluation: You describe that almost a 10\% of patients in both groups died before the first imaging evaluation. This percentage is comparable to the hyperprogression rate in patients treated with check-point inhibitors. Did these patients exhibit any kind of specific profile? Did they have any kind of adverse event, such as (for instance) diarrhea? Please, add a comment in this regard.

We thank the reviewer for the interesting parallel with the hyperprogression. We analysed the characteristics of the patients who died before the first imaging evaluation. A sizeable proportion of this population had a Child-Pugh B class at baseline (24.1\%). Other traditionally negative predictors of survival were also relatively prevalent in this population, such as PS $=1(48.3 \%)$ and AFP $>400 \mathrm{ng} / \mathrm{ml}(41.4 \%)$. Instead, the rate of AEs was lower in comparison with the remaining population (dermatological AEs $13.8 \%$, diarrhea 13.8\%, hypertension 17.2\%). This pattern could be explained by the fact that these patients simply did not live enough to develop AEs. Also, in the case of dermatological AEs, the lack of these manifestations can be seen as a further negative prognostic factor. We added these considerations in the appropriate section,

10) Permanent sorafenib discontinuation: Did you evaluate the type of progression in patients who progressed? If so, is this pattern similar between the two groups? Different patterns of progression may explain different outcomes in the groups.

We thank the reviewer for suggesting to analyse another potential confounding factor. We were able to assess the progression pattern as described by Reig et al in the 198 patients who had a documented radiological progression (227 total progressors minus 29 patients who died before performing a follow up imaging). The rates of BCLCp-B, BCLCp-C1 and BCLp-C2 were comparable across the two groups. We added he following information:

METHODS - Permanent discontinuation of sorafenib: "In patients who discontinued sorafenib for progression, the impact of the pattern of progression [REIG] on the post-progression survival was also analysed. Post progression survival was defined as the time from the last dose of sorafenib and death."

RESULTS - Permanent discontinuation of sorafenib: "For the 198 patients who had a documented radiological progression at the time of sorafenib discontinuation, the pattern of progression was similar (BCLCP-B 26.2 vs 26.3\%; BCLCp-C1 39.3 vs 38.6\%; BCLCp-C2 34.5 vs $35.1 \%$ ) in Group A and $B$, respectively $(p=0.994)$."

DISCUSSION - "Second, in a similar line, the duration of treatment and the median daily dose were the only significantly different variables in the follow-up of the two groups, thus excluding an imbalance in other confounding factors (for instance, a difference in the pattern of progression)"

11) Permanent sorafenib discontinuation: How did you define sorafenib intolerance?

Sorafenib intolerance was defined as the presence of unmanageable grade 2-4 AEs not responding to dose reductions and/or temporary interruption of treatment. This definition was also used in the referenced paper by lavarone and colleagues (Hepatology 2015). We added this information under the METHODS - Permanent discontinuation of sorafenib session. 
The lack of a statistically significant correlation between extrahepatic spread and overall survival has been further investigated. At the univariate analysis, the median OS was 13.5 and 9.9 months for non-metastatic and metastatic patients respectively. The survival curves of patients with and without extrahepatic spread, however, crossed at about 30 months due to a relatively slower slope of the curve of metastatic patients. In its turn, this finding reflected the presence of a subgroup of patients with limited extrahepatic spread who achieved a long-lasting radiological disease control (about $18 \%$ of all metastatic patients). We added the following considerations to the Results session:

"Extrahepatic spread did not reach the full statistical significance as a predictor of OS in our series $(p=0.111)$. The lack of a significant result was due to the presence of a subgroup of patients with limited extrahepatic spread (18\% of all metastatic patients) who achieved a long-lasting radiological disease control."

13) OS evaluation and correlates: Did you try to introduce the pattern of progression in regression analyses? It could be also useful for a better understanding of the results.

We thank the reviewer for this useful suggestion. We performed an additional survival analysis to assess the relationship between the pattern of progression and the post-progression survival. This analysis included the 198 patients who had a documented radiological progression (227 total progressors minus 29 patients who died before performing a follow up imaging). We found significantly different post progression survival for the $B C L C p-B, B C L C p-C 1$ and $B C L C p-C 2$ patterns, thus validating the prognostic ability of the BCLC upon progression classification. We added the following lines:

RESULTS: The pattern of progression was significantly correlated with the post-progression survival. The median OS was 11.0 [95\% CI 7.4-14.8], 6.0 [95\% Cl 4.2-7.6], and 4.7 [95\% Cl 3.4-6.2] months in the BCLCp-B, BCLCp-C1 and BCLCp-C2 classes, respectively ( $p<0.001)$. Progression due to new extrahepatic lesion(s) was associated with a significantly worse post-progression survival compared to other patterns of progression $(4.9$ [95\% $\mathrm{Cl} 3.1$ 6.5] vs 8.0 [95\% Cl 5.6-10.0]months, $p<0.001)$.

14) OS evaluation and correlates: Did you consider including the number of previous TACE procedures (obviously in those patients treated with TACE) in the regression analyses? We have great evidence supporting a "quick" migration to systemic treatment if TACE fails, and we are probably doing so nowadays. However, it is highly likely that patients in the group A received more TACE sessions than those in group B. These first patients could have been at higher risk of liver decompensation, leading to a shorter period of time under systemic treatment and have shorter OS. Please, add a comment in this regard.

We are grateful to the reviewer for pointing out this possible confounder. We fully agree that an early migration is a key element to rescue the risk of liver function decompensation and let the patients have the greatest likelihood of receiving the maximum survival benefit from the systemic treatments. Actually, the participating centers had been always skeptical about the benefit of countless TACE treatments even in the immediate presorafenib era, due to previous experience of the low likelihood of tumor control and the parallel increase in the risk of deocmpensation. As a consequence, no significant differences in the number of previous TACE procedures were found in the two groups. In particular, the rate of patients who had received more than 2 TACE was relatively low (20-25\% in both groups). These findings reflected into the rate of patients who discontinued sorafenib due to liver decompensation, who was also very similar in the two groups. The description of the number of TACE was now added to Table 1.

Moreover, as suggested by the reviewer, we performed a subgroup analysis including the patients who had received at least one TACE. Two multivariate regressions were created, using different cut-offs to identify a possible detrimental role of repeated TACE. The results of these analyses supported the importance of a quick migration to systemic treatments and have been added in the following paragraph:

"Results - Subgroup analyses - Patients previously treated with trans-arterial chemoembolization (TACE): Overall, 58.4 and $55.4 \%$ of patients of the Group A and Group B had received at least TACE before the first dose of sorafenib. As reported in Table 1, there was no difference in the number of $\operatorname{TACE}(p=0.270)$. To investigate a possible detrimental effect of repeated TACE, we created two different multivariate regressions which included only the subgroup of patients which received at least one TACE.

In the first model, we set 2 TACE as a cut-off (1-2 vs 3 or more TACE). In this case, the number of previous TACE met a borderline significance at the univariate analysis $(p=0.083)$ and was not confirmed as an independent predictor of survival at the multivariate Cox regression $(p=0272)$.

In the second model, the cut-off was set at 3 TACE (1-3 vs 4 or more TACE). In this model, the number of previous procedures still met a borderline significance at the univariate analysis $(p=0.056)$, but this time was confirmed as a prognosticator at the multivariate regression $(\mathrm{HR} 1.595,95 \% \mathrm{Cl}$ $1.065-2.390, p=0.024)$.

Notably, the period of treatment was confirmed as an independent predictor of survival both in the first (HR $0.703,95 \% \mathrm{Cl} 0.513-0.963, p=0.028)$ and in the second model (HR 0.693,95\% Cl 0.506-0.949, $p=0.022$ ) despite the reduced statistical power of the subgroup analyses." 
15) In the legend, you mention that you are describing median and IQR. However, it looks like the values that you are describing are standard deviations. I recommend checking it.

The interquartile range is now correctly reported in the Table.

FIGURE

The use of mRECIST overestimates complete response (and objective response). In my opinion, it would be a good idea reporting objective response using RECIST rather than mRECIST.

The Figure now reports the partial response and complete response rates according to RECIST 1.1 


\title{
IMPROVED MANAGEMENT OF ADVERSE EVENTS WITH TAILORED SORAFENIB DOSING PROLONGS SURVIVAL OF HEPATOCELLULAR CARCINOMA PATIENTS
}

Francesco Tovoli ${ }^{1}$, Luca lelasi ${ }^{1}$, Andrea Casadei-Gardini ${ }^{2-3}$, Alessandro Granito ${ }^{1}$, Francesco Giuseppe Foschi ${ }^{4}$, Giulia Rovesti ${ }^{3}$, Giulia Negrini ${ }^{1}$, Giulia Orsi ${ }^{3}$, Matteo Renzulli ${ }^{5}$, Fabio Piscaglia ${ }^{1}$

1. Department of Medical and Surgical Sciences, University of Bologna, Bologna, Italy.

2. Department of Medical Oncology, Istituto Scientifico Romagnolo per Lo Studio e Cura Dei Tumori (IRST) IRCCS, Meldola, Italy.

3. Department of Oncology and Haematology, University Hospital of Modena, Modena, Italy.

4. Department of Internal Medicine, Degli Infermi Hospital, Faenza, Italy.

5. Unit of Radiology, Department of Diagnostic Medicine and Prevention, Sant'Orsola Hospital, University of Bologna, Bologna, Italy

\author{
Corresponding Author \\ Francesco Tovoli, MD \\ Department of Medical and Surgical Sciences, University of Bologna \\ Via Massarenti 9, 40136 Bologna, Italy \\ Phone number : +390512142214 , Fax number +390516362725 \\ E-mail: francesco.tovoli2@unibo.it
}

Keywords: hepatocellular carcinoma; sorafenib; adverse events; outcome; prognosis; learning curve.

Electronic word count: 6649

Number of Figures: 4 Number of Tables: 6 (1 supplementary)

Conflict of interests: $\quad$ FT and AG: consultant for Bayer AG

ACG: consultant for Bayer, advisory board for Eisai

FP: consultant for Astrazeneca, Bayer AG, EISAI, GE, Tiziana life sciences; Speaker bureau honoraria: Bayer AG, Bracco, EISAI, Laforce; research contract with Esaote LI, FGF, GR, GN, GO, and MR: none to declare.

Financial support: none to declare

Authors contribution: Study concept and design: FT, AG, LI;

Data collection: LI, ACG, AG, FGF;

Experiments and procedures: GR, GO, GN, MR;

Writing and critical revision of the draft: FT, ACG, FP. 


\section{ABSTRACT}

Background\&Aims. Sorafenib is associated with multiple adverse events (AEs), potentially causing its permanent interruption. The impact of the physicians experience on the management of these AEs and the relative implications on clinical outcomes are unknown. We verified if the AEs management changed over time and if these modifications impacted on treatment duration and overall survival (OS).

Methods. We analysed the prospectively collected data of 338 consecutive patients who started sorafenib between January 2008 and December 2017 in three tertiary care centres in Italy. Patients were divided according to the starting date: Group A (2008-2012; n=154), and Group B (2013-2017, n=184). Baseline and follow up data were compared. In the OS analysis, patients who received second-line treatments were censored when starting the new therapy.

Results. Baseline characteristics, AEs, and radiological response were consistent across groups. Patients in Group B received a lower median daily dose (425 vs $568 \mathrm{mg} /$ day, $\mathrm{p}<0.001$ ) due to more frequent dose modifications. However, treatment duration was longer (5.8 vs 4.1 months, $p=0.021$ ) with a trend toward a higher cumulative dose in Group B. Notably, the OS was also higher (12.0 vs 11.0 months, $p=0.003$ ) with a sharp increase in the 2-year survival rate ( 28.1 vs $18.4 \%, p=0.003)$ in Group B. The multivariate timedependent Cox regression confirmed later period of treatment as an independent predictor of survival (HR $0.728,95 \% \mathrm{Cl} 0.581-0.937, \mathrm{p}=0.013)$. Unconsidered confounders were unlikely to affect these results at the sensitivity analysis.

Conclusions: experience in the management of sorafenib-related AEs prolongs treatment duration and survival. This factor should be considered in the design of future randomised clinical trials including a sorafenib treatment arm, as an underestimate of sample size may derive.

Lay summary. Sorafenib is the standard frontline systemic treatment for hepatocellular carcinoma since over a decade. Its tolerability is limited by different adverse events which might lead to its permanent discontinuation in sizeable proportion of patients. After a careful analysis of potential confounders, we demonstrated that the physicians' experience in managing adverse events related to sorafenib has improved overtime, with longer treatment periods and less permanent discontinuation for toxicities. More importantly, these improvements also translated into a longer survival of the patients. Our results have relevant repercussions in clinical practice and in the design of future clinical trials. 


\section{INTRODUCTION}

Sorafenib is a multitarget tyrosine kinase inhibitor (TKI) currently used for the treatment of hepatocellular carcinoma (HCC) not amenable to surgery or locoregional treatments [1]. Sorafenib significantly prolongs patients' overall survival (OS), but its use is associated with different adverse events (AEs), mainly dermatological, gastrointestinal and cardiovascular $[2,3]$. The management of these AEs can require dose reductions and temporary interruptions. In a sizeable proportion of patients, however, these modifications are not able to avoid intolerable or severe AEs, resulting in permanent drug discontinuation [1]. It might seem common sense that the experience accumulated in the prescription of sorafenib could lead to improved management of its related AEs. However, the exact impact of the operators' experience on the prescribing patterns of sorafenib has been investigated rarely [4]. Similarly, it is not known whether this phenomenon can lead to an increase in OS. The latter point is of crucial importance, as dermatological AEs have been demonstrated to have a favourable prognostic impact $[5,6]$. Thus, avoiding a definitive suspension of sorafenib in these patients would be extremely beneficial. Moreover, data from observational studies $[7,8]$ and randomised clinical trials (RCTs) [9] indirectly seem to suggest that the OS of patients treated with sorafenib is progressively increasing. However, the reasons for this phenomenon have not been fully elucidated. A very recent monocentric study [10] suggested that the experience in managing sorafenib AEs has improved overtime, but rigorous multicentric studies taking into account also timedependent variables, addressing the possible confounding factor of second-line treatments, and providing a confirmation of the survival benefit through multivariable regressions models are still lacking.

The primary objective of our study is to verify whether the treatment schedules of sorafenib (in terms of average daily dose, duration of treatment, cumulative dose) and the percentage of discontinued patients for intolerable AE varied over time. The secondary objective was to verify if these differences had a correlation with the OS after corrections for confounders.

\section{METHODS}

\section{Design of the study}

The present study was performed using the medical records from the databases of Sant'Orsola-Malpighi Hospital, University of Bologna, Italy; Istituto Scientifico Romagnolo per lo Studio e la Cura dei Tumori (IRST) IRCCS, Meldola, Italy; and Degli Infermi Faenza Hospital, Italy. All of these databases include prospectively collected consecutive patients prescribed with sorafenib and followed up. Data were entered every 3-6 months into electronic data files by co-investigators from each centre taking part and checked at the data management centre for internal consistency. For the purposes of this study, we retrospectively considered patients who were prescribed from January 2008 to December 2017. The starting date coincides with the 
licensing of sorafenib in Italy, and therefore with the possibility of prescription by all of the study centres.

The closing date preceded the licensing of regorafenib in Italy by one year and was chosen to allow an adequate follow-up of the patients. For the purpose of this study, the whole 2008-2017 timeframe was divided in two exactly equally long periods (January 2008 - December 2012 and January 2013 - December 2017). The closing time for last follow up was 31 March 2019.

\section{Baseline evaluation}

We recorded the following data for each patient at the time of the first prescription of sorafenib: time elapsed since the first diagnosis of HCC, previous treatments for HCC, aetiology of the underlying liver disease, presence/absence of liver cirrhosis, and comorbidities. The main parameters entailing the residual liver function according to the Child-Pugh score were also registered. Finally, we included data about the tumour staging according to the Barcelona Clinic for Liver Cancer (BCLC) classification. The baseline values of alfa-fetoprotein (AFP) were also available for all patients. Patients with HCV-related cirrhosis in sustained virological response after a viral eradication with direct anti-viral agents were considered a group of special interest, as recent reports suggested that they might develop a more aggressive form of HCC [11].

\section{$\underline{\text { Sorafenib prescription }}$}

All patients were prescribed with sorafenib at the initial dose of $400 \mathrm{mg}$ twice a day. At the time of the first prescription of sorafenib, all patients were provided with a diary and instructed about the possible sorafenib-related AEs to allow early recognition and treatment. A preventive application of urea cream $20 \%$ on hands and feet twice a day, plus loperamide as an over-the-counter medication in case of diarrhoea were recommended. Patients were advised to measure blood pressure every day and to contact their respective centres in case of new symptoms.

\section{$\underline{\text { Adverse events evaluation and management }}$}

New symptoms arising after the treatment start, as well as their timing, were thoroughly recorded. The medical records included both a brief description of each AE as well as its coding according to the Common Terminology Criteria for Adverse Events (CTCAE) used at the time of registration. Since the CTCAE version was upgraded from version 3.0 to version 4.03 in June 2010, all of the AEs occurred before that date were re-codified according to the newer version to allow a correct comparison between groups. Dose modifications in response to the AEs (including dose reductions and dose stops) were performed according to the manufacturer's recommendations. When sorafenib became available in 2008 , the dose modifications were performed in strict accordance with the instruction contained in the SHARP protocol [2]. As a result, dose stops were extremely limited, and dose reductions below the minimum recommended dose of 400 mg once daily were rare, to aggressively reach the maximum tolerated dose in every single patient at every single moment. Patients who had recurring unmanageable toxicities at the dose of $400 \mathrm{mg}$ once daily were 
almost invariably discontinued for intolerance. As the experience increased, this approach became less rigid and more patient-tailored. The main aim shifted to identify for each patient a regimen with limited toxicities but a higher likelihood of being tolerated on a longer term. For instance, in selected cases, very short dose stops (1-2 days) were performed even for Grade 2 toxicities, provided that: a) the patient perceived them as extremely limiting their quality of life; b) the physician regarded these toxicities as a potential threat to the compliance of the patient. Also, if AEs reappeared even after a 7-day dose stop and a subsequent dose reduction to $400 \mathrm{mg}$ once daily, we became more prone at performing a further dose reduction to $200 \mathrm{mg}$ once daily rather than permanently discontinue sorafenib. This new focus was supported by cumulative evidence favouring a tailored approach $[12,13]$ and discouraging an aggressive treatment re-escalation [14].

\section{Biochemistry and imaging evaluations}

As a general rule, under normal conditions, biochemistry was reassessed every 2 weeks during the first 8 weeks of treatment, then every 4 weeks. The first imaging evaluation of response was scheduled 8-10 weeks after the first dose of sorafenib, with the subsequent radiological controls scheduled every 12 weeks. Computed tomography of thorax-abdomen-pelvis with iodinated contrast medium was the preferred imaging technique. For patients with contraindications to the iodinated contrast medium, a magnetic resonance imaging of the abdomen paired with a high-resolution computed chest tomography was performed. Radiologic evaluation of response during follow-up was done by CT-scan according to the Response Evaluation Criteria In Solid Tumours (RECIST) v1.1 [15]. These criteria were preferred over the modified RECIST (mRECIST) $[16,17]$ due to the possible overestimation of tumor necrosis in the setting of systemic treatments by the latter criteria [18].

\section{$\underline{\text { Permanent discontinuation of sorafenib }}$}

Sorafenib was continued until 1) radiological and clinical progression (for patients eligible for second-line clinical trials, radiological progression alone was considered a sufficient reason for discontinuation); 2) unacceptable AEs, or; 3 ) deterioration of liver function. Categorisation into these classes was performed according to the same criteria previously proposed by lavarone and colleagues [19]. In particular, sorafenib intolerance was defined as the presence of unmanageable grade 2-4 AEs not responding to dose reductions and/or temporary interruption of treatment [19]. In patients who discontinued sorafenib for progression, the impact of the pattern of progression [20] on the post-progression survival was also analysed. Post progression survival was defined as the time from the last dose of sorafenib to death.

\section{$\underline{\text { OS evaluation and correlates }}$}

OS was measured from the date of starting sorafenib until the date of death. Patients who received a second-line treatment in the setting of clinical trials were censored at the time of the first dose of the new 
therapy). The rate of long-survivors, defined as patients with an OS $\geq 24$ months $[7,17]$, was considered a

variable of particular interest. Twelve- and 24-month landmark analyses were performed to further evaluate the long-term modifications of OS. The choice of these cut-offs derived from the most recently described median OS of sorafenib-treated patients in the REFLECT trial [9] and by the recently proposed cut off to define long survivors to sorafenib $[7,21]$, respectively. The same cut-off were used in the landmark analyses of a recent ancillary study of the REFLECT trial [22]. To address the possible bias deriving from more second-line RCTs being available in the latest years (resulting in a higher number of censored patients in one group), a completeness quantification of the follow up was performed. Also, a sensitivity analysis was performed to evaluate how strong an unmeasured confounder would have to be to disprove the possibly observed relationship between the period of treatment and OS.

\section{$\underline{\text { Ethics }}$}

The study protocol was reviewed and approved by the local Ethics Committees (Bologna Authority Hospital Ethic Committee for Bologna centre and Romagna Ethic Committee for Meldola and Faenza centres, respectively). All patients gave their written informed consent according to the Ethics Committees' recommendations. The study was conducted according to the ethical guidelines of the 1975 Declaration of Helsinki.

\section{$\underline{\text { Statistics }}$}

Continuous variables are expressed as median and interquartile range. Categorical variables are expressed as frequencies. Group comparisons were performed with the Mann-Whitney test. Categorical variables were evaluated using two-tailed Fisher's test. Survival curves were estimated using the product-limit method of Kaplan-Meier. The role of stratification factors was analysed with log-rank tests. To define the predictors of OS we used a time-dependent covariates survival approach including statistically significant clinical variables $(p<0.05)$ from the univariate Cox analysis. The completeness quantification of the follow up was performed according to the C-index, as proposed by Clark et al. [23]. Briefly, C-index is the ratio of the total observed follow-up years divided by the total potential follow-up years. In the case of our study, the observed follow-up for each patient was the time from study entry to the death or censoring. The potential follow-up for each patient was the time from study entry to the closing date of the study or patient death, whichever came first. The sensitivity analysis was performed calculating the E-value and the limit of its $95 \%$ confidence interval $(\mathrm{Cl})$ closest to the null $[24,25]$. Statistical analyses were performed using SPSS version 23.0 (SPSS Inc., Chicago, IL, USA) (Supplementary CTAT Table).

\section{RESULTS}

$\underline{\text { Study population }}$ 
This study included 338 patients. Most patients were cirrhotic (96.8\%), viral hepatitis was the leading cause of their chronic liver disease (HBV infection: 21,8\%, HCV infection 49.7\%, nonviral causes $28.5 \%$ ). One hundred fifty-four patients started sorafenib in the 2008-2012 period (Group A), while the remaining 184 patients began treatment in the 2013-2017 period (Group B).

\section{Baseline characteristics}

There were no significant differences in the baseline characteristics between the two groups, especially in terms of demographics, liver function, and tumour staging (TABLE 1). Amongst patients with HCV-related cirrhosis, the rate of HCV-RNA positivity was $87.6 \%$ and $85.9 \%$ in Groups A and B, respectively ( $p=0.748$ ). Amongst patients with HCV-related liver disease, the rate of HCV-RNA positive patients was 87.6 and $85.9 \%$ in Groups A and B, respectively ( $p=0.748$ ). These proportions accounted for 19 and 26 patients in sustained virological response in the first and second period, respectively. All of the 19 patients in the 2008-2012 period had achieved a sustained virological response after interferon-based treatments. Amongst the 26 patients who started sorafenib in the 2013-2017 period, 18 had eradicated the HCV infection with interferon-based treatments and 8 with second-generation direct antiviral agents. No patients with HBVrelated cirrhosis were HBV-DNA positive at the time of sorafenib prescription. Finally, we found a similar rate of comorbities in the two groups (SUPPLEMENTARY TABLE 1).

\section{Follow up}

The median follow-up was 10.5 months (range 0.2-78.9), with no significant differences between study groups (10.3 vs 10.5 months in Groups A and B, respectively). The C-index was $94.0 \%$ in Group A and 85.1\% in Group B.

\section{$\underline{\text { Adverse events evaluation and management }}$}

In both groups, almost every patient reported at least one AE after sorafenib start (TABLE 2). Overall, the rate of patients reporting at least one dermatological AE (hand-foot skin reaction or skin rash) was similar in the two groups $(34.4 \%$ vs $38.0 \%$ in groups $A$ and $B$, respectively, $p=0.498$ ). The rate of patients complaining of gastrointestinal symptoms was also similar in the two groups ( $42.9 \%$ vs $50.5 \%, p=0.189$ ). The prevalence of treatment-related arterial hypertension was $24.8 \%$ in Group A and $27.8 \%$ in Group B $(p=0.538)$.

In most patients, the management of AEs required at least one dose modification. The rate of patients who required a dose reduction to $400 \mathrm{mg} /$ day was higher in Group B than in Group A (86.4 vs 75.3\%, p=0.009). Re-escalation to a higher dose, however, was possible for a sizeable number of patients in both groups. (TABLE 3) More patients received a dose reduction to 200 mg/day in Group B compared to Group A (30.4 vs 
$13.6 \%, \mathrm{p}<0.001)$. In this case, the majority of patients tolerated a dose escalation to $400 \mathrm{mg} /$ day. This refers

to the first dose adjustment and not to the number of dose adjustments during follow-up.

A similar number of patients received at least one temporary dose stop ( $87.5 \mathrm{vs} 81.2 \%, p=0.130$ ). However, the rate of patients who received a temporary dose stop in at least two different occurrences was higher in Group B compared to Group A ( 54.3 vs $42.2 \%, p=0.029)$.

$\underline{\text { Imaging evaluation }}$

Fourteen (9.1\%) and $15(8.2 \%)$ patients died before the first imaging follow up in Groups A and B, respectively, in a clinical setting of early progression $(p=0.846)$. A sizeable proportion of this population had a Child-Pugh B class at the baseline (24.1\%). Other traditionally negative predictors of survival were also relatively prevalent in this population, such as PS=1 (48.3\%) and AFP>400 ng/ml (41.4\%). Instead, the rate of AEs was lower in comparison with the remaining population (dermatological AEs 13.8\%, diarrhea $13.8 \%$, hypertension $17.2 \%)$. After the first imaging evaluation, the objective response and the disease control rates were comparable across the study groups (FIGURE 1). In particular, the disease control rate was $51.3 \%$ in Group A and $56.5 \%$ in Group B ( $p=0.688)$.

\section{Permanent sorafenib discontinuation}

During the follow-up, sorafenib was permanently discontinued in all but 4 patients, all of them belonging to the Group B. The median treatment duration was significantly higher in Group B (5.8 vs 4.1 months, $\mathrm{p}=0.021$ ), with a lower median daily dose ( $425 \mathrm{vs} 568 \mathrm{mg} /$ day, $\mathrm{p}<0.001$ ) compared to Group A. There was a trend toward a higher cumulative dose in the Group B, which however did not reach the statistical significance (75.0 vs $65.6 \mathrm{~g}, \mathrm{p}=0.313$ ). Progression was the main cause leading to permanent sorafenib discontinuation in both groups (TABLE 4). Of note, permanent discontinuation due to AEs was significantly higher in Group A compared to Group B (20.8\% vs 9.2\%, p<0.001). For the 198 patients who had a documented radiological progression at the time of sorafenib discontinuation, the pattern of progression was similar (BCLCp-B 26.2 vs 26.3\%; BCLCp-C1 39.3 vs 38.6\%; BCLCp-C2 34.5 vs 35.1\%) in Group A and B, respectively $(p=0.994)$.

\section{$\underline{\text { OS evaluation and correlates }}$}

The multivariable analysis of factors related to the OS included all clinically statistically significant variables at the univariate analysis, considered for a forward stepwise approach. The multivariable analysis consistently identified Child-Pugh class, performance status, macrovascular invasion, alfa-fetoprotein $\geq 400$ $\mathrm{ng} / \mathrm{ml}$, the appearance of dermatological AEs and period of treatment (HR $0.728,95 \% \mathrm{Cl} 0.581-0.937$, $p=0.013$ ) as independent predictors of OS (TABLE 5). Extrahepatic spread did not reach the full statistical significance as a predictor of OS in our series $(p=0.111)$. The lack of a significant result was due to the 
presence of a subgroup of patients with limited extrahepatic spread ( $18 \%$ of all metastatic patients) who

achieved a long-lasting radiological disease control.

When dividing the patients according to their era of treatment, the median OS was significantly higher in Group B compared to Group A (12.0 vs 11.0 months, $p=0.002$ ) (FIGURE 2). Notably, the rate of long survivors was significantly higher in the Group B than in Group A, with a 2-year and 3-year survival rate of 28.1 vs $18.4 \%(p=0.003)$ and 14.6 vs $6.8 \%(p=0.037)$, respectively. The landmark analysis largely confirmed these findings (FIGURE 3). Analysing the patients according to their radiological response, the survival gain was particularly evident for patients who achieved disease control ( 21.5 vs 19.5 months, $p=0.009$ ). On the contrary, OS did not differ across the study groups for patients with progressive disease at their first imaging control (FIGURE 4).

The sensitivity analysis reported an E-value of 1.356 for the association between period of treatment and OS. The limit of the $95 \%$ confidence interval closest to the null hypothesis was 1.335 . These values mean that the observed association between OS and period of treatment could be nullified only by an unmeasured confounder associated with both OS and period, with a strength similar to that of performance status or AFP $>400 \mathrm{ng} / \mathrm{ml}$, which is very unlikely to have been missed. On the contrary, a weaker confounder could not disprove this association.

\section{Subgroup analyses}

- Patients previously treated with direct antiviral agents

With the limitations of the reduced sample size $(n=8)$, patients with post-DAA HCC had a more frequent onset directly in the advanced stage (75.0\%), but their response to sorafenib was not worse compared to the remaining study population (disease control rate 62.5\%). Also, the overall survival of these patients was not significantly impaired (median 12.0 months).

- Patients previously treated with trans-arterial chemoembolisation (TACE)

Overall, 58.4 and $55.4 \%$ of patients of the Group A and Group B had received at least TACE before the first dose of sorafenib. As reported in Table 1 , there was no difference in the number of TACE $(p=0.270)$. To investigate a possible detrimental effect of repeated TACE, we created two different multivariate regressions which included only the subgroup of patients which received at least one TACE.

In the first model, we set 2 TACE as a cut-off (1-2 vs 3 or more TACE). In this case, the number of previous TACE met a borderline significance at the univariate analysis $(p=0.083)$ and was not confirmed as an independent predictor of survival at the multivariate Cox regression $(p=0272)$. 
In the second model, the cut-off was set at 3 TACE (1-3 vs 4 or more TACE). In this model, the number of

previous procedures still met a borderline significance at the univariate analysis $(p=0.056)$, but this time was confirmed as a prognosticator at the multivariate regression ( $\mathrm{HR} 1.595,95 \% \mathrm{Cl} 1.065-2.390, \mathrm{p}=0.024$ ).

Notably, the period of treatment was confirmed as an independent predictor of survival both in the first ( $\mathrm{HR} 0.703,95 \% \mathrm{Cl} 0.513-0.963, \mathrm{p}=0.028$ ) and in the second model ( $\mathrm{HR} 0.693,95 \% \mathrm{Cl} 0.506-0.949, \mathrm{p}=0.022$ ) despite the reduced statistical power of the subgroup analyses.

- Pattern of progression and post-progression survival

The pattern of progression was significantly correlated with the post-progression survival. The median OS was 11.0 [95\% Cl 7.4-14.8], 6.0 [95\% Cl 4.2-7.6], and 4.7 [95\% Cl 3.4-6.2] months in the BCLCp-B, BCLCp-C1 and $B C L C p-C 2$ classes, respectively $(p<0.001)$. Progression due to new extrahepatic lesion(s) was associated with a significantly worse post-progression survival compared to other patterns of progression $(4.9[95 \% \mathrm{Cl}$ 3.1-6.5] vs 8.0 [95\% Cl 5.6-10.0]months, $\mathrm{p}<0.001)$.

\section{DISCUSSION}

We provide direct evidence supporting two novel findings. First, the management of sorafenib-related AEs has changed over time, now allowing a longer duration of treatment. Second and most important, these changes also translate into a survival gain.

Regarding the first point, our study provides the first direct confirmation of previous indirect evidence. In the registrative Phase 3 SHARP trial [2], 38\% of patients had to discontinue sorafenib due to intolerable AEs. Subsequent observational studies which enrolled populations in the first four years following sorafenib licensing (and therefore comparable to Group A of our study) showed a comparable rate of discontinuation for toxicities. For example, in a large sorafenib field practice study including 296 patients recruited in 20082010 , this rate was about $40 \%$ [26]. In their prospective study on the role of dermatological AEs, Reig et al. [5] reported a withdrawal rate for AEs of 30.5\% amongst 147 patients enrolled in the 2008-2011 period. This rate was slightly lower (21\%) in the real-life GIDEON study (comprising 3371 patients enrolled in 20092012) [8]. On the other hand, another large multicenter observational study (INSIGHT) evaluated patients enrolled up to 2014 and described a sensibly lower rate of patients with unmanageable intolerance (15.5\%) [7]. This trend found further indirect confirmation from the second-line clinical trials of ramucirumab (REACH-2) and pembrolizumab (KEYNOTE-224). The rate of patients entering these trials following sorafenib withdrawal for AES was 17.1 and $20.0 \%$, respectively [27,28]. A final clue came from the noninferiority Phase 3 RCT lenvatinib vs sorafenib (REFLECT), in which only $7 \%$ of patients in the sorafenib treatment arm had to discontinue the drug due to AEs [9]. 
In addition to the confirmation of this indirect evidence, our study also showed that a reduced interruption

rate for AEs corresponds to an extension of the treatment duration. This longer treatment duration in the second period is even more striking if we take into the account the possible interference derived by the absence of approved drugs and the relative paucity of clinical trials in the first period of the study. This different clinical setting, in fact, could have lead to longer treatment durations in the first period, as sorafenib could not have been discontinued to offer second line options, as they were lacking at that time. This data was not inferable from previous observational studies, nor from the comparison of the SHARP and REFLECT data. While treatment beyond radiological progression was allowed in the first trial, progression automatically resulted in permanent treatment discontinuation as of protocol rules in the latter [2,9]. Only very recently, Raoul and colleagues [10] reported similar results in a retrospective monocenter study. Their report of an increase in treatment duration from 4.3 months in $2008-2012$ vs 5.9 months in $2013-2017$ is strikingly similar to our results. Our study provides a validation of these initial results through a large multicenter collaboration. Of note, more flexible and personalised management of treatment scheduled also resulted in a lower median daily dose. This finding should not be seen as a justification to start treatment at a half dose or to be particularly indulgent with dose reductions and stops. Instead, it was the result of accumulating experience, which led to specific choices under well-defined circumstances, performed to balance the highest tolerable dose with the highest likelihood of achieving long term compliance. These choices were possible only thanks to a close follow up after sorafenib initiation, with easy access to unscheduled visits and consultations to detect AEs, manage them promptly, and adjust the dosage. Only in this scenario, it is possible to conjugate treatment compliance with optimal efficacy, without unneeded treatment interruptions or cancellations [5].

Most importantly, we demonstrated for the first time that experience in the management of AEs also has an impact on OS. While the median gain may seem modest (about one month), it should also be considered that the whole study population also included early progressors, i.e. patients for which improvement in the management of AEs is unlikely to mirror into a survival gain (as confirmed by our subgroup analysis). On the contrary, the median survival gain was doubled (about two months) in patients who achieved disease control. The difference in the rate of long survivors was even more striking. It can, therefore, be hypothesised that the improved management of $\mathrm{AEs}$ in responders patients reduced the risk of unnecessary drug discontinuation allowing a more extended treatment and a longer OS. So far there had been no direct demonstration of an improvement in the OS of patients treated with sorafenib over time. In the aforementioned study by Raoul et al. [10], the OS was in fact significantly higher in the last five years (12 vs 8 months) in the univariate analysis, but it was not possible to confirm this result also in a multivariate regression. Even indirect evidence about the critical aspect of OS is minimal, deriving mainly from information collected in the REFLECT trial. In this study, the median OS in the sorafenib treatment arm was remarkably superior to that initially reported in the SHARP trial (12.4 vs 10.7 months). However, the 
REFLECT study excluded patients with unfavourable prognostic factors (neoplastic occupation of the liver>

$50 \%$, neoplastic thrombosis of the main portal trunk, biliary invasion) and this choice could at least partially justify the apparent improvement of the OS. As a consequence, no reliable information about OS modifications was available before our study.

We are aware that analysing a possible period-effect can be tricky due to multiple possible analytical pitfalls and confounders. However, we have reasonable evidence to believe that our results were genuine. First, the baseline characteristics of the patients were similar in the two groups in terms of tumour burden, liver function, performance status, comorbidities, and number of previous TACE procedures, making the hypothesis of an improved referral of the patients unlikely as a possible alternative explanation. Second, in a similar line, the duration of treatment and the median daily dose were the only significantly different variables in the follow-up of the two groups, thus excluding an imbalance in other confounding factors (for instance, a difference in the pattern of progression). Third, censoring the survival of patients who received second-line treatments excluded the longer OS was justified by improvements in oncological treatments. While this choice caused a slightly lower completeness of the follow up in the second group, the completeness remained very high in absolute terms. Fourth, innovations in non-oncological therapies are an equally unlikely alternative explanation, as demonstrated by both the unmodified OS in non-responder patients and the similar rate of HCV viraemic patients. In this regard, the 8 patients previously treated with direct antiviral agents had a more frequent onset in the advanced stage as previously described by Reig at al [11], but their response to sorafenib was not different from that of the remaining study population. Finally, any theoretical confounder not considered in the previous points should have required a very relevant impact on OS to disprove our results, as evidenced by sensitivity analysis.

In conclusion, the increasing experience with the management of sorafenib-related AEs is leading to longer treatment duration and survival in responder patients. Our results have immediate implications, both in clinical and research terms. About clinical implications, a lower discontinuation rate for AEs implies better access to subsequent treatments. Regorafenib, for example, is only available for patients who progressed to sorafenib. At the same time, the improved management of AEs is extremely likely to translate also into optimised management of other TKIs (regorafenib, cabozantinib) with a cumulative OS advantage. As for research implications, the increasing median OS has obvious implications in the design of RCTs in which sorafenib is used as a comparator drug. In this case, an OS estimate based on the SHARP trial or early observational studies could lead to an underestimation of the sample size needed to reach a superiority or non-inferiority endpoint. The most immediate examples are the numerous trials of immune checkpoint inhibitors versus sorafenib in the frontline systemic setting [29]. These trials will be of critical importance in shaping the future therapeutic algorithms for the systemic treatment of HCC in the next future, and their results are eagerly awaited. Therefore, we suggest that the increased survival under sorafenib should be 
considered in the design of future trials, to minimise the risk of unintentional failure due to an underestimated sample size.

\section{REFERENCE}

1. Forner A, Da Fonseca LG, Díaz-González A, Sanduzzi-Zamparelli M, Reig M, Bruix J. Controversies in the management of hepatocellular carcinoma. JHEP Reports, 2019 [in press] doi: 10.1016/j.jhepr.2019.02.003

2. Llovet JM, Ricci S, Mazzaferro V, Hilgard P, Gane E, Blanc JF, de Oliveira AC, Santoro A, Raoul JL, Forner A, Schwartz M, Porta C, Zeuzem S, Bolondi L, Greten TF, Galle PR, Seitz JF, Borbath I, Häussinger D, Giannaris T, Shan M, Moscovici M, Voliotis D, Bruix J; SHARP Investigators Study Group. Sorafenib in advanced hepatocellular carcinoma. N Engl J Med. 2008; 359(4): 378-90.

3. Cheng AL, Kang YK, Chen Z, Tsao CJ, Qin S, Kim JS, Luo R, Feng J, Ye S, Yang TS, Xu J, Sun Y, Liang H, Liu J, Wang J, Tak WY, Pan H, Burock K, Zou J, Voliotis D, Guan Z. Efficacy and safety of sorafenib in patients in the Asia-Pacific region with advanced hepatocellular carcinoma: a phase III randomised, double-blind, placebocontrolled trial. Lancet Oncol. 2009; 10(1): 25-34.

4. Casadei Gardini A, Scarpi E, Foschi FG, Marisi G, Maltoni M, Frassineti GL. Impact of physician experience and multidisciplinary team on clinical outcome in patients receiving sorafenib. Clin Res Hepatol Gastroenterol. 2018 [in press] doi: 10.1016/j.clinre.2018.11.005

5. Reig M, Torres F, Rodriguez-Lope C, Forner A, LLarch N, Rimola J, Darnell A, Ríos J, Ayuso C, Bruix J. Early dermatologic adverse events predict better outcome in HCC patients treated with sorafenib. J Hepatol. $2014 ; 61(2): 318-24$.

6. Díaz-González Á, Sanduzzi-Zamparelli M, Sapena V, Torres F, LLarch N, Iserte G, Forner A, da Fonseca L, Ríos J, Bruix J, Reig M.. Systematic review with meta-analysis: the critical role of dermatological events in patients with hepatocellular carcinoma treated with sorafenib. Aliment Pharmacol Ther. 2019; 49(5): 48291.

7. Ganten TM, Stauber RE, Schott E, Malfertheiner P, Buder R, Galle PR, Göhler T, Walther M, Koschny R, Gerken G.. Sorafenib in Patients with Hepatocellular Carcinoma-Results of the Observational INSIGHT Study. Clin Cancer Res. 2017; 23(19): 5720-8

8. Marrero JA, Kudo M, Venook AP, Ye SL, Bronowicki JP, Chen XP, Dagher L, Furuse J, Geschwind JH, de Guevara LL, Papandreou C, Takayama T, Sanyal AJ, Yoon SK, Nakajima K, Lehr R, Heldner S, Lencioni R. Observational registry of sorafenib use in clinical practice across Child-Pugh subgroups: The GIDEON study. J Hepatol. 2016; 65(6): 1140-7 
9. Kudo M, Finn RS, Qin S, Han KH, Ikeda K, Piscaglia F, Baron A, Park JW, Han G, Jassem J, Blanc JF, Vogel A, Komov D, Evans TRJ, Lopez C, Dutcus C, Guo M, Saito K, Kraljevic S, Tamai T, Ren M, Cheng AL. Lenvatinib versus sorafenib in first-line treatment of patients with unresectable hepatocellular carcinoma: a randomised phase 3 non-inferiority trial. Lancet. 2018; 391(10126): 1163-73.

10. Raoul JL, Adhoute X, Penaranda G, Perrier H, Castellani P, Oules V, Bourlière M.Sorafenib: Experience and Better Management of Side Effects Improve Overall Survival in Hepatocellular Carcinoma Patients: A Real-Life Retrospective Analysis. Liver Cancer 2019 [in press] doi: 10.1159/000497161.

11. Reig M, Mariño Z, Perelló C, Iñarrairaegui M, Ribeiro A, Lens S, Díaz A, Vilana R, Darnell A, Varela M, Sangro B, Calleja JL, Forns X, Bruix J. Unexpected high rate of early tumor recurrence in patients with HCVrelated HCC undergoing interferon-free therapy. J Hepatol. 2016 ;65(4): 719-726

12. Abbadessa G, Rimassa L, Pressiani T, Carrillo-Infante C, Cucchi E, Santoro A. Optimized management of advanced hepatocellular carcinoma: four long-lasting responses to sorafenib. World J Gastroenterol. 2011; 17(19): 2450-3.

13. Brose MS, Frenette CT, Keefe SM, Stein SM. Management of sorafenib-related adverse events: a clinician's perspective. Semin Oncol. 2014; 41 Suppl 2: S1-16.

14. Rimassa L, Pressiani T, Boni C, Carnaghi C, Rota Caremoli E, Fagiuoli S, Foa P, Salvagni S, Cortesi E, Chiara Tronconi M, Personeni N, Bozzarelli S, Chiara Banzi M, Fanello S, Romano Lutman F, Giordano L, Santoro A. A phase II randomized dose escalation trial of sorafenib in patients with advanced hepatocellular carcinoma.Oncologist. 2013; 18(4): 379-80.

15. Eisenhauer EA, Therasse P, Bogaerts J, Schwartz LH, Sargent D, Ford R, et al. New response evaluation criteria in solid tumours: revised RECIST guideline (version 1.1). Eur J Cancer. 2009; 45: 228-47

16. Llovet JM, Di Bisceglie AM, Bruix J, Kramer BS, Lencioni R, Zhu AX, et al, Design and endpoints of clinical trials in hepatocellular carcinoma. J Natl Cancer Inst. 2008; 100: 698-711

17. Lencioni R, Llovet, JM. Modified RECIST (mRECIST) assessment for hepatocellular carcinoma. Semin Liver Dis. 2010; 30: 52-60

18. Bruix J, Reig M, Sangro B.Assessment of treatment efficacy in hepatocellular carcinoma: Response rate, delay in progression or none of them. J Hepatol. 2017 Jun;66(6):1114-1117

19. lavarone M, Cabibbo G, Biolato M, Della Corte C, Maida M, Barbara M, Basso M, Vavassori S, Craxì A, Grieco A, Cammà C, Colombo M. Predictors of survival in patients with advanced hepatocellular carcinoma who permanently discontinued sorafenib. Hepatology. 2015; 62(3): 784-91. 
20. Reig M, Rimola J, Torres F, Darnell A, Rodriguez-Lope C, Forner A, Llarch N, Ríos J, Ayuso C, Bruix J.

Postprogression survival of patients with advanced hepatocellular carcinoma: rationale for second-line trial design. Hepatology. 2013;58(6): 2023-31.

21. Sacco R, Granito A, Bargellini I, Zolfino T, Saitta C, Marzi L, Tapete G, Bresci G, Marinelli S, Tovoli F, Attardo S, Rossi M, Urbani L, Marchi S, Buccianti P, Cabibbo G. Clinical outcomes with long-term sorafenib treatment of patients with hepatocellular carcinoma: a multicenter real-life study. Future Oncol. 2018; 14(29): 3049-58.

22. Kudo M, Finn RS, Qin S, Han KH, Ikeda K, Cheng AL, et al. Analysis of survival and objective response (OR) in patients with hepatocellular carcinoma in a phase III study of lenvatinib (REFLECT).J Clin Oncol 37, 2019 (suppl 4; abstr 186).

23. Clark TG, Altman DG, De Stavola BL. Quantification of the completeness of follow-up. Lancet. 2002; 359(9314): 1309-10.

24. Ding P, VanderWeele TJ. Sensitivity analysis without assumptions.Epidemiology 2016;27:368-77.

25. VanderWeele TJ, Ding P. Sensitivity Analysis in Observational Research: Introducing the E-Value. Ann Intern Med. 2017; 167(4): 268-74.

26. lavarone M, Cabibbo G, Piscaglia F, Zavaglia C, Grieco A, Villa E, Cammà C, Colombo M; SOFIA (SOraFenib Italian Assessment) study group. Field-practice study of sorafenib therapy for hepatocellular carcinoma: a prospective multicenter study in Italy. Hepatology. 2011; 54(6): 2055-63.

27. Zhu AX, Kang YK, Yen CJ, Finn RS, Galle PR, Llovet JM, Assenat E, Brandi G, Pracht M, Lim HY, Rau KM, Motomura K, Ohno I, Merle P, Daniele B, Shin DB, Gerken G, Borg C, Hiriart JB, Okusaka T, Morimoto M, Hsu Y, Abada PB, Kudo M; REACH-2 study investigators. Ramucirumab after sorafenib in patients with advanced hepatocellular carcinoma and increased a-fetoprotein concentrations (REACH-2): a randomised, double-blind, placebo-controlled, phase 3 trial. Lancet Oncol. 2019 ; 20(2): 282-296.

28. Zhu AX, Finn RS, Edeline J, Cattan S, Ogasawara S, Palmer D, Verslype C, Zagonel V, Fartoux L, Vogel A, Sarker D, Verset G, Chan SL, Knox J, Daniele B, Webber AL, Ebbinghaus SW, Ma J, Siegel AB, Cheng AL, Kudo M; KEYNOTE-224 investigators. Pembrolizumab in patients with advanced hepatocellular carcinoma previously treated with sorafenib (KEYNOTE-224): a non-randomised, open-label phase 2 trial. Lancet Oncol. 2018; 19(7): 940-52.

29. Tovoli F, Casadei-Gardini A, Benevento F, Piscaglia F. Immunotherapy for hepatocellular carcinoma: A review of potential new drugs based on ongoing clinical studies as of 2019. Dig Liver Dis. 2019 [in press]. doi: 10.1016. 
TABLE 1. Baseline characteristics of study patients according the period of start of sorafenib. Continuous variables are expressed as median (interquartile range). Categorical variables are reported as frequencies (percentage)

\begin{tabular}{lllc} 
Variables & $\begin{array}{c}\mathbf{2 0 0 8 - 2 0 1 2} \\
\text { period }(\mathbf{n = 1 5 4})\end{array}$ & $\begin{array}{c}\mathbf{2 0 1 3}-\mathbf{2 0 1 7} \\
\text { period }(\mathbf{n = 1 8 4})\end{array}$ & $\mathbf{P}$ \\
\hline Age (years) & $70(62-75)$ & $69(58-75)$ & 0.334 \\
Males & $135(87.7)$ & $156(84.3)$ & 0.426 \\
Cirrhosis & $149(96.8)$ & $178(96.7)$ & 1.000 \\
Etiology & & & \\
- HBV & $37(24.0)$ & $36(19.6)$ & 0.354 \\
- HCV & $75(48.7)$ & $93(50.5)$ & 0.745 \\
- Nonviral & $42(27.3)$ & $55(29.9)$ & 0.630
\end{tabular}

\section{Disease duration}

$-<6$ months

$-6-12$ months

24 (14.9)

31 (15.8)

0.199

- > 12 months

84 (53.9)

$83(44.0)$

Bilirubin (mg/dl)

$0.91(0.62-1.26)$

$0.91(0.64-1.20)$

0.885

Albumin (g/L)

36 (33-39)

$36(33-40)$

0.566

INR (ratio)

1.14 (1.06-1.25)

$1.13(1.06-1.23)$

0.438

Ascites

$13(8.4)$

$18(9.8)$

0.709

Encephalopathy

6 (3.9)

$4(2.2)$

0.522

Child-Pugh B

11 (7.1)

16 (8.7)

0.690

Tumor stage

- Intermediate (BCLC B) 54 (35.1)

$72(38.2)$

0.498

- Advanced (BCLC C)

100 (64.9)

$112(61.8)$

Performance status

- PS 0

98 (63.6)

130(70.7)

0.200

$$
\text { -PS } 1
$$

56 (36.4)

54 (29.3)

Macrovascular invasion

50 (32.5)

$50(27.2)$

0.339

Extrahepatic spread

$51(33.1)$

76 (41.3)

0.143

AFP $>400 \mathrm{ng} / \mathrm{ml}$

47 (30.5)

45 (24.5)

0.222

Number of previous

TACE procedures 


\begin{tabular}{|c|c|c|c|}
\hline - None & $64(41.6)$ & $82(44.6)$ & \\
\hline - One & $24(15.6)$ & $32(17.4)$ & \\
\hline - Two & $28(18.2)$ & $32(17.4)$ & 0.270 \\
\hline - Three & $20(13.0)$ & $18(9.8)$ & \\
\hline - Four & $11(7.1)$ & $16(8.7)$ & \\
\hline - Five or more & $7(4.5)$ & $4(2.2)$ & \\
\hline
\end{tabular}

HBV: hepatitis B virus; HCV: hepatitis C virus; BCLC: Barcelona Clinic for Liver Cancer; AFP: alfa-fetoprotein;

TACE: transarterial chemoembolisation. 
TABLE 2. Prevalence of treatment-emergent adverse events (AEs) according to the period of start of sorafenib. Data are expressed as frequencies (percentage).

\begin{tabular}{|c|c|c|c|c|}
\hline \multirow{2}{*}{ Adverse Events (AEs) } & \multicolumn{2}{|c|}{$\begin{array}{c}2008-2012 \text { period } \\
(n=154)\end{array}$} & \multicolumn{2}{|c|}{$\begin{array}{c}2013-2017 \text { period } \\
(n=184)\end{array}$} \\
\hline & All grades & Grade $\geq 3$ & All grades & Grade $\geq 3$ \\
\hline Any $A E$ & $151(98.0)$ & $56(34.4)$ & $181(98.1)$ & $61(33.2)$ \\
\hline Fatigue & $96(62.4)$ & $18(11.9)$ & $133(72.2)$ & $12(6.4)$ \\
\hline Diarrhea & 61 (39.6) & 9 (5.9) & $86(46.7)$ & $7(3.7)$ \\
\hline Hand foot skin reaction & $45(29.2)$ & $6(3.9)$ & $63(34.3)$ & $14(7.4)$ \\
\hline Hypertension & $38(24.8)$ & $10(6.5)$ & $51(27.8)$ & $10(5.5)$ \\
\hline Skin rash & $15(9.7)$ & $3(1.9)$ & $19(10.3)$ & $7(3.8)$ \\
\hline Weight loss & $15(9.7)$ & 0 & $17(9.2)$ & $2(0.9)$ \\
\hline Hyporexia & $13(8.4)$ & 0 & $10(5.4)$ & 0 \\
\hline Nausea & $11(7.1)$ & $2(1.3)$ & $17(9.2)$ & $2(0.9)$ \\
\hline Thrombocytopenia & $9(5.8)$ & $2(1.3)$ & $13(7.1)$ & 0 \\
\hline Alopecia & $8(5.2)$ & NA & $10(5.4)$ & NA \\
\hline Anaemia & $7(4.5)$ & $2(1.3)$ & $5(2.7)$ & 0 \\
\hline Leucopenia & $6(3.9)$ & $2(1.3)$ & $8(4.3)$ & $2(0.9)$ \\
\hline Dysphonia & $6(3.9)$ & 0 & $7(3.8)$ & $2(0.9)$ \\
\hline Mucositis & $5(3.2)$ & 0 & $5(2.7)$ & 0 \\
\hline Epistaxis & $2(1.3)$ & 0 & $4(2.2)$ & $1(0.5)$ \\
\hline
\end{tabular}


TABLE 3. Dose modification strategies in response to the treatment-emergent adverse events. Data are

1

2

3

4

5

6

7

8

9

10

11

12

13

14

15

16

17

18

19

20

21

22

23

24

25

26

27

28

29

30

31

32

33

34

35

36

37

38

39

40

41

42

43

44

45

46

47

48

49

50

51

52

53

54

55

56

57

58

59

60

61

62

63

64

65

expressed as frequencies (percentage).

\begin{tabular}{llll} 
& $\begin{array}{c}2008-\mathbf{2 0 1 2} \\
\text { period }(\mathrm{n}=\mathbf{1 5 4})\end{array}$ & $\begin{array}{c}\mathbf{2 0 1 3}-\mathbf{2 0 1 7} \\
\text { period }(\mathrm{n}=\mathbf{1 8 4})\end{array}$ & P \\
\hline Dose reduction to $400 \mathrm{mg} /$ day & $113(75.3)$ & $157(86.4)$ & 0.009 \\
- Re-escalation to $800 \mathrm{mg} /$ day & $34 / 113(30.2)$ & $35 / 157(22.0)$ & 0.341 \\
Dose reduction to $200 \mathrm{mg} /$ day & $21(18.2)$ & $56(35.2)$ & $<0.001$ \\
- Re-escalation to $400 \mathrm{mg} /$ day & $15 / 21(71.4)$ & $34 / 56(60.7)$ & 0.691
\end{tabular}


TABLE 4. Reasons leading to sorafenib permanent discontinuation

1

2

* Four patients still receiving sorafenib in this group

$\begin{array}{ll}129(71.7) & 0.127 \\ 17(9.4) & <0.001 \\ 26(14.4) & 0.752 \\ 8(4.4) & 0.557\end{array}$


TABLE 5. Predictors of survival according to the multivariable Cox regression performed using appearance of dermatological adverse events as a time-dependent variable.

\begin{tabular}{|c|c|c|c|c|c|c|}
\hline \multicolumn{3}{|c|}{ Univariate analysis } & Variable & \multicolumn{3}{|c|}{ Multivariate analysis } \\
\hline HR & $95 \% \mathrm{Cl}$ & $\mathrm{P}$ & & $\mathrm{HR}$ & $95 \% \mathrm{Cl}$ & $P$ \\
\hline 1.190 & 0.834-1.699 & 0.337 & Age $>70$ years & - & - & - \\
\hline 1.034 & $0.815-1.311$ & 0.784 & Male gender & - & - & - \\
\hline 1.011 & $0.722-1.300$ & 0.733 & Viral etiology & - & - & - \\
\hline & & & Time since first HCC diagnosis > & & & \\
\hline 0.888 & $0.789-1.000$ & 0.049 & 12 months & - & - & 0.723 \\
\hline 2.638 & $1.751-3.972$ & $<0.001$ & Child Pugh B status & 2.093 & $1.364-3.211$ & 0.001 \\
\hline 1.651 & $1.287-2.118$ & $<0.001$ & Performance status (0 vs 1 ) & 1.357 & $1.043-1.765$ & 0.023 \\
\hline 1.770 & $1.373-2.282$ & $<0.001$ & Macrovascular invasion & 1.722 & $1.331-2.622$ & $<0.001$ \\
\hline 1.250 & $0.989-1.584$ & 0.095 & Extrahepatic spread & - & - & 0.111 \\
\hline 1.486 & $1.147-1.924$ & 0.003 & Alfa-fetoprotein >400 ng/ml & 1.328 & $1.024-1.724$ & 0.033 \\
\hline 0.562 & $0.436-0.725$ & $<0.001$ & $\begin{array}{l}\text { Dermatological AEs } \\
\text { Period of treatment }\end{array}$ & 0.624 & $0.482-0.808$ & $<0.001$ \\
\hline 0.694 & $0.547-0.880$ & 0.003 & (2013-2017 vs 2008-2012) & 0.728 & $0.581-0.937$ & 0.013 \\
\hline
\end{tabular}


FIGURE 1. Radiological response according to the Response Evaluation Criteria in Solid Tumors version 1.1

1

2

3

4

5

6

7

8

9

10

11

12

13

14

15

16

17

18

19

20

21

22

23

24

25

26

27

28

29

30

31

32

33

34

35

36

37

38

39

40

41

42

43

44

45

46

47

48

49

50

51

52

53

54

55

56

57

58

59

60

61

62

63

64

65

at the first imaging follow-up (performed 8-10 weeks after the start of sorafenib)

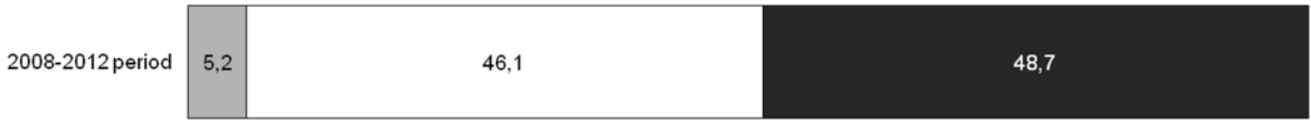

1,1

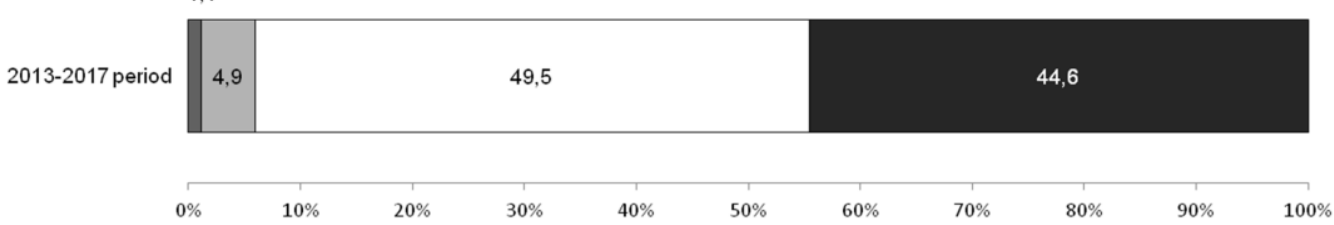

-Complete response aPartial response

$\square$ Stable disease

- Progressive disease

$P=0.688$ 
FIGURE 2. Overall survival outcomes

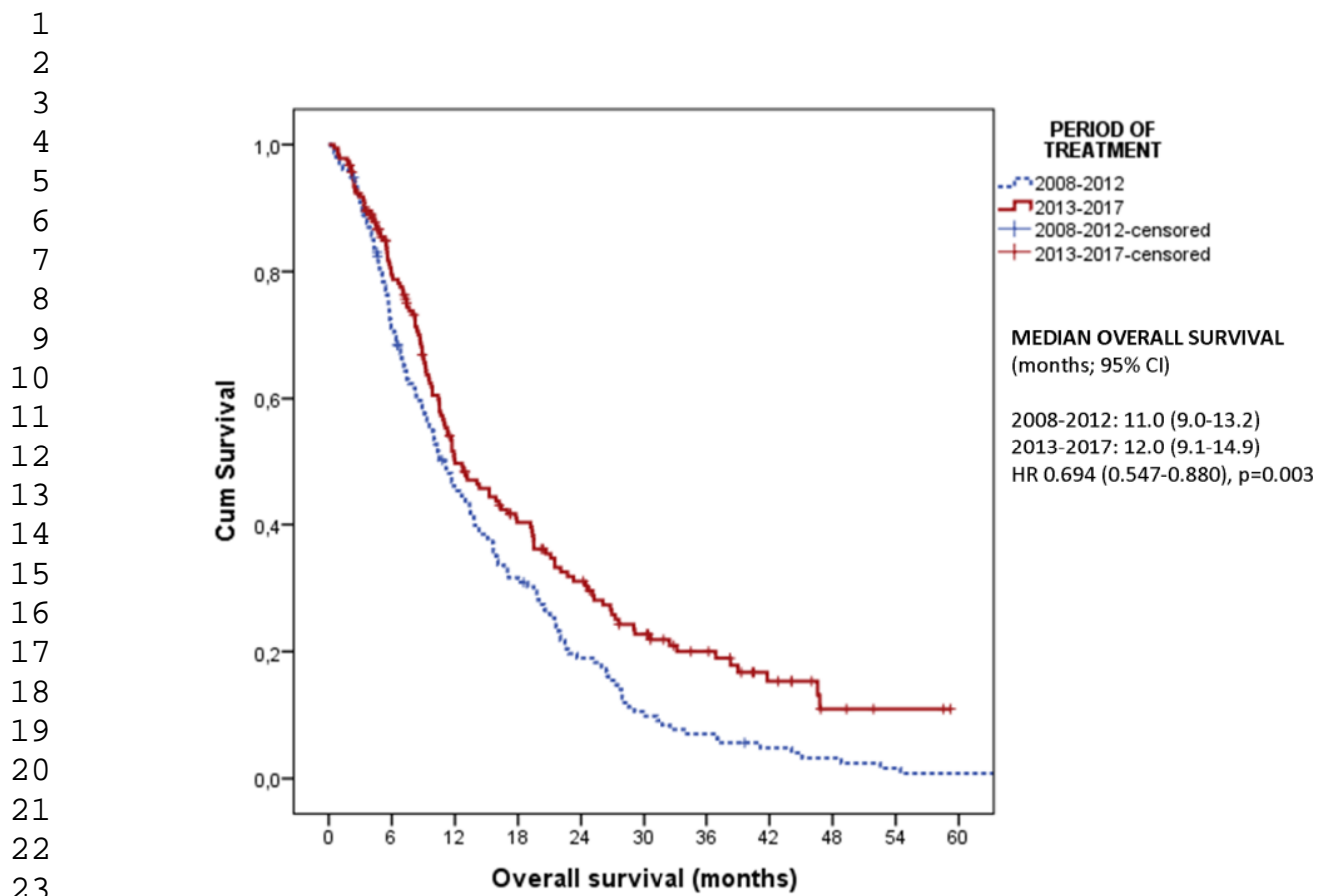


A

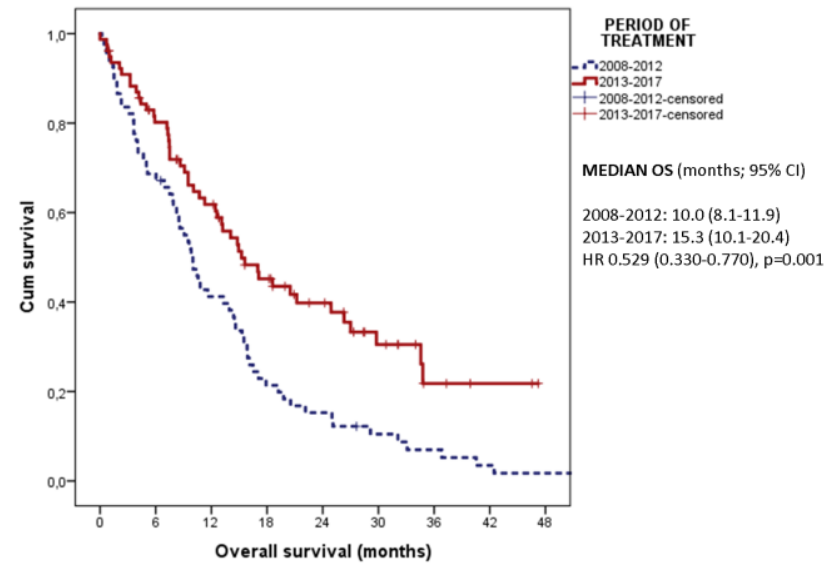

B

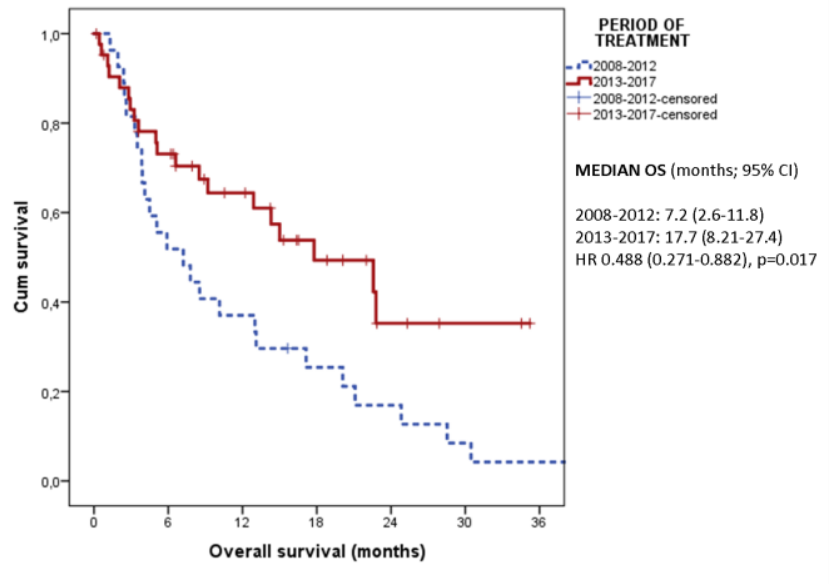

FIGURE 3. Overall survival (OS) outcomes according to the 12-month (A) and 24-month landmark analyses 
FIGURE 4. Overall survival (OS) outcomes stratified according to the first imaging response in progressors (A) and responders (B).

A

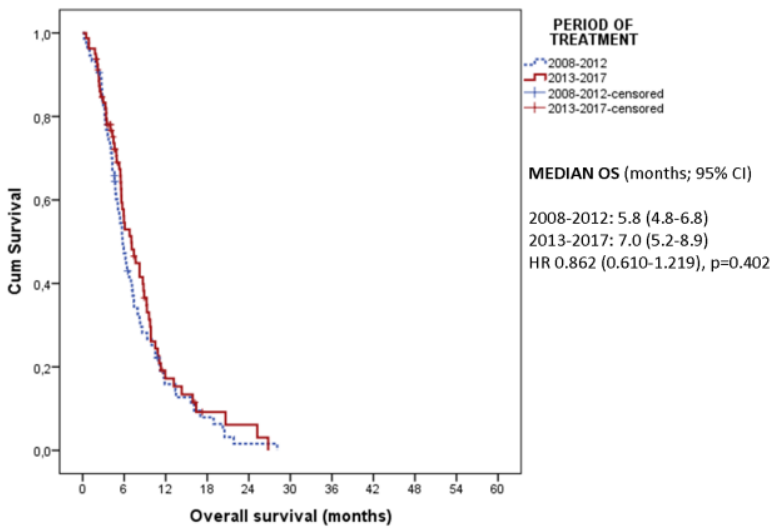

B

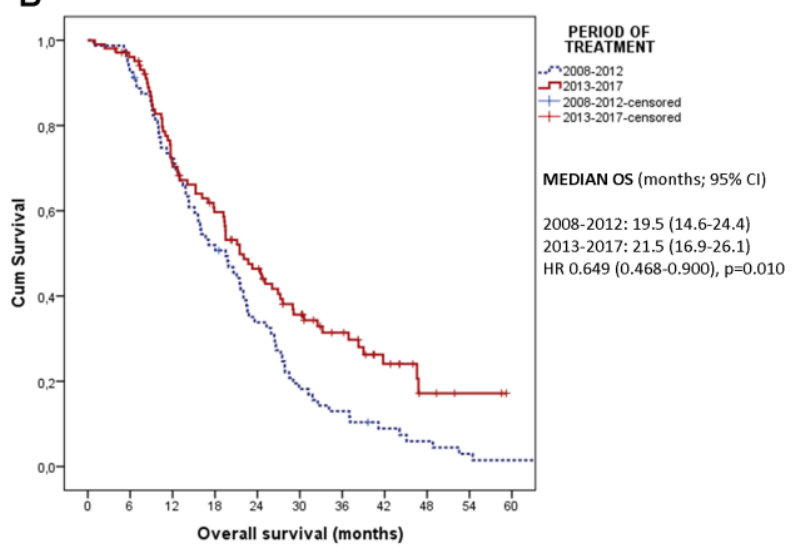




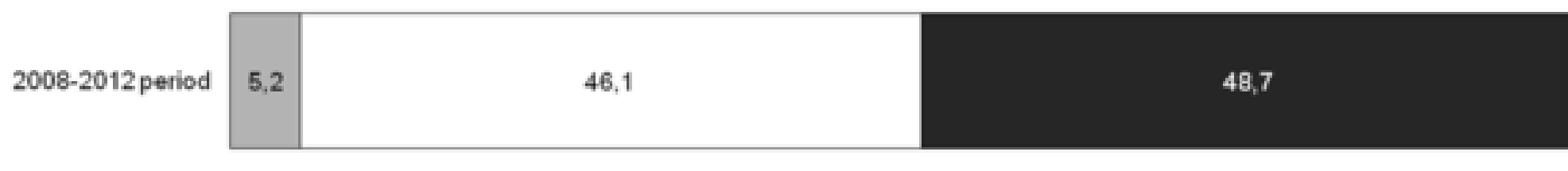

1,1

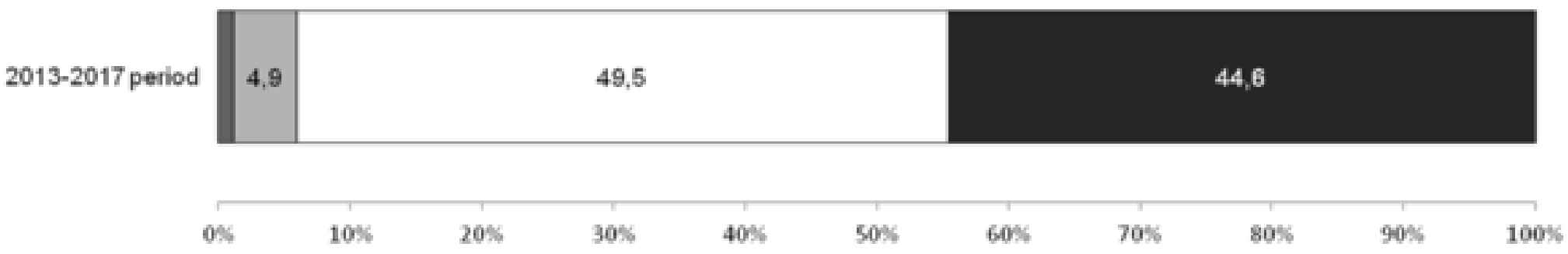

aComplete response

口Partial response

口Stable disease

- Progressive disease

$P=0.688$ 


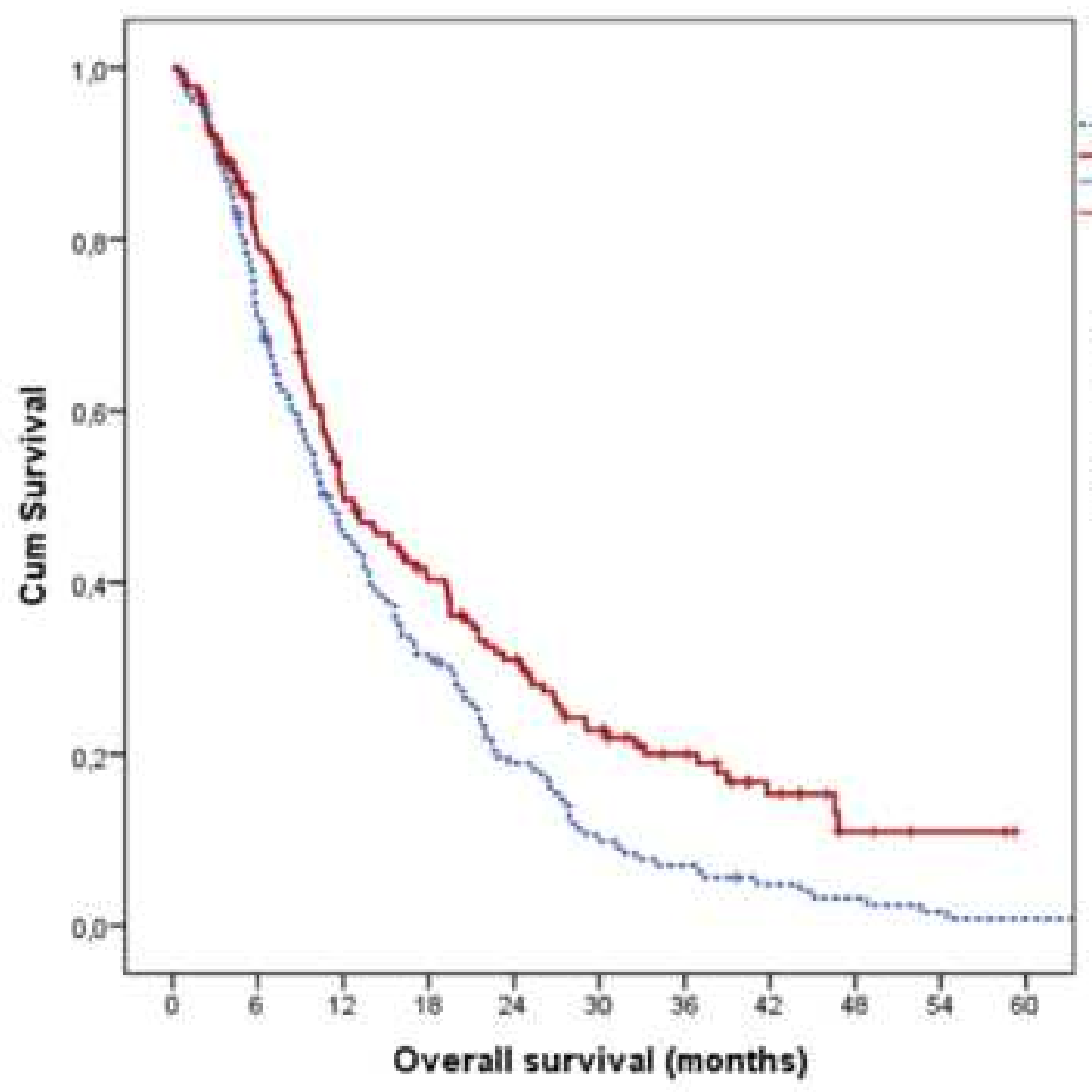

PEPIOD OF
IREATMENT

$\sim 2008-2012$

-72013-2017

-2008-2012-censcred

+2013-2017-censored

MEDIAN OVERALL SURVIVAL

(months: 95\% Cl)

2008-2012: $11.0(9.0-13.2)$

2013-2017: 12.0 (9.1-14.9)

HR $0.694(0.547-0.880), p=0.003$ 
A

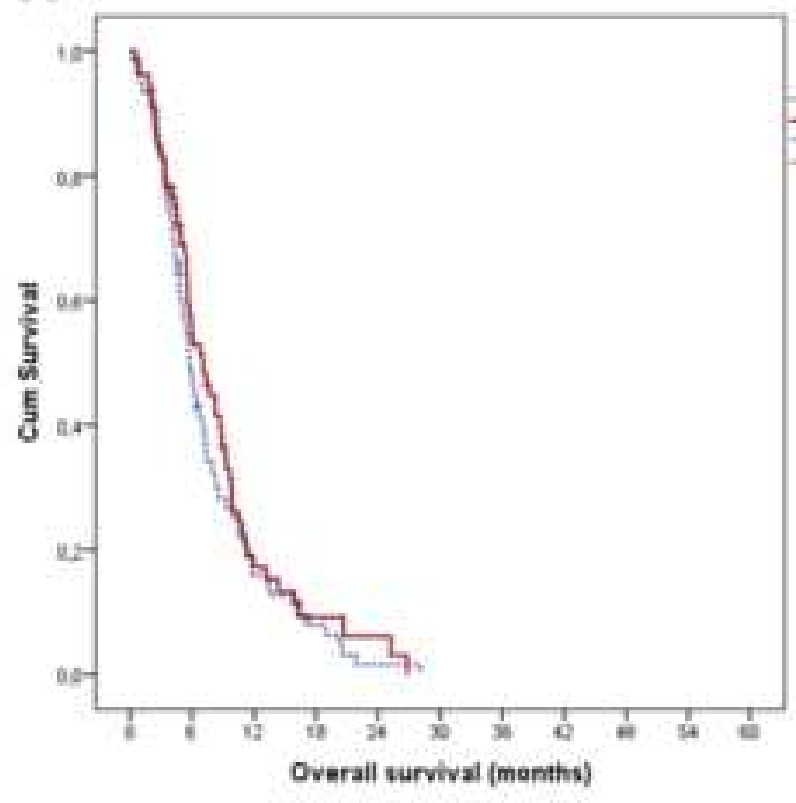

B

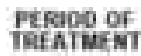

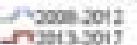

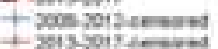

MEDUAN OS (maeths; 55\% CI)

$2003.2012: 5.8$ (4.11-6.8)

$2013.2017: 70(5.2 .89)$

หค 0.\$62 (0.610-1.219), $\rho=0.40)$

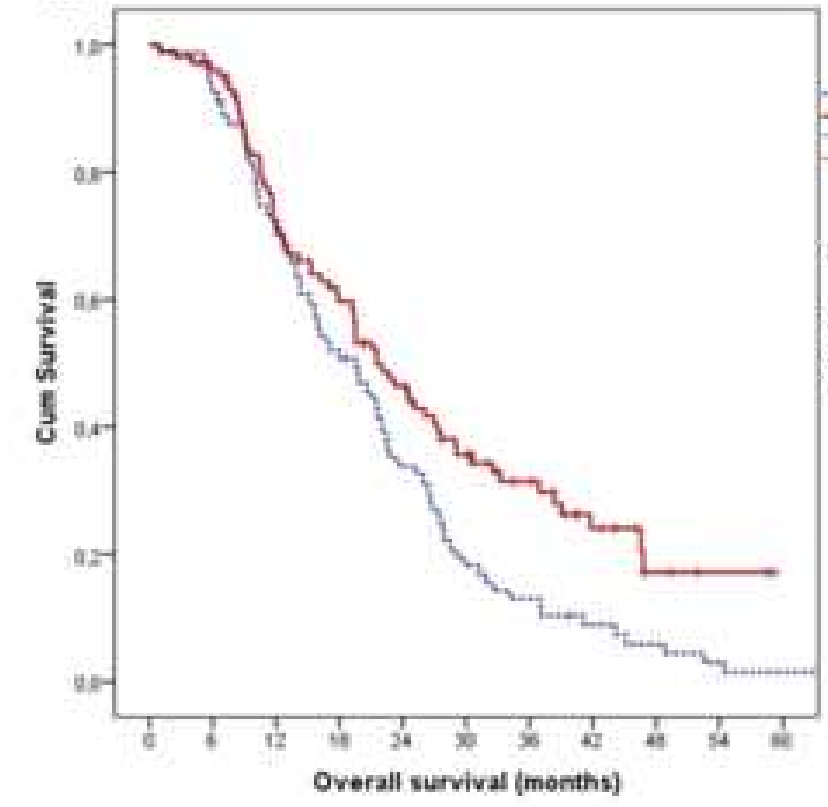

\section{Preapo of}

רo00:012

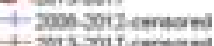

MEDIAN OS (months; 95\% a)

2006-2012: $195(14.6-24,4)$

2013.2017: $21.5(15.9-26.1)$

HR 0.649 (0.468-0.900), $p=0.010$ 
A

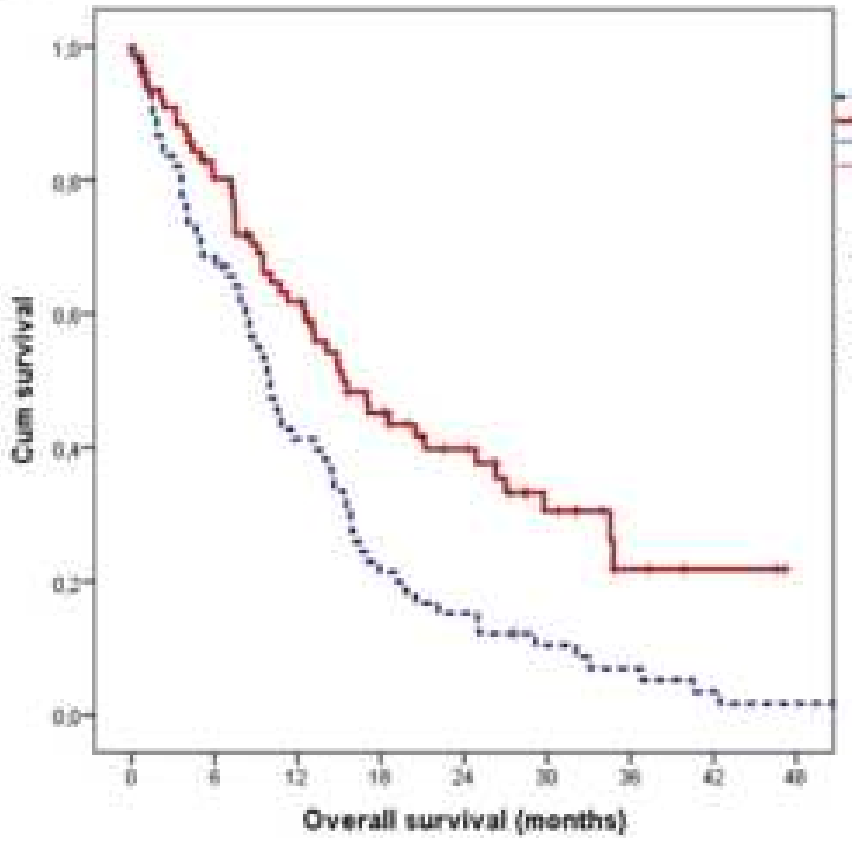

B

PEAOD of
TREATMENT

nosestis

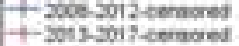

MEDIAN os imonths: $95 \mathrm{~N}$ c)

$2000-2012 \div 10.0(8.1-11.9)$

2013-2017: $15.3(10.120 .4)$

in 0.529 j0.330-0.7not p=0.001

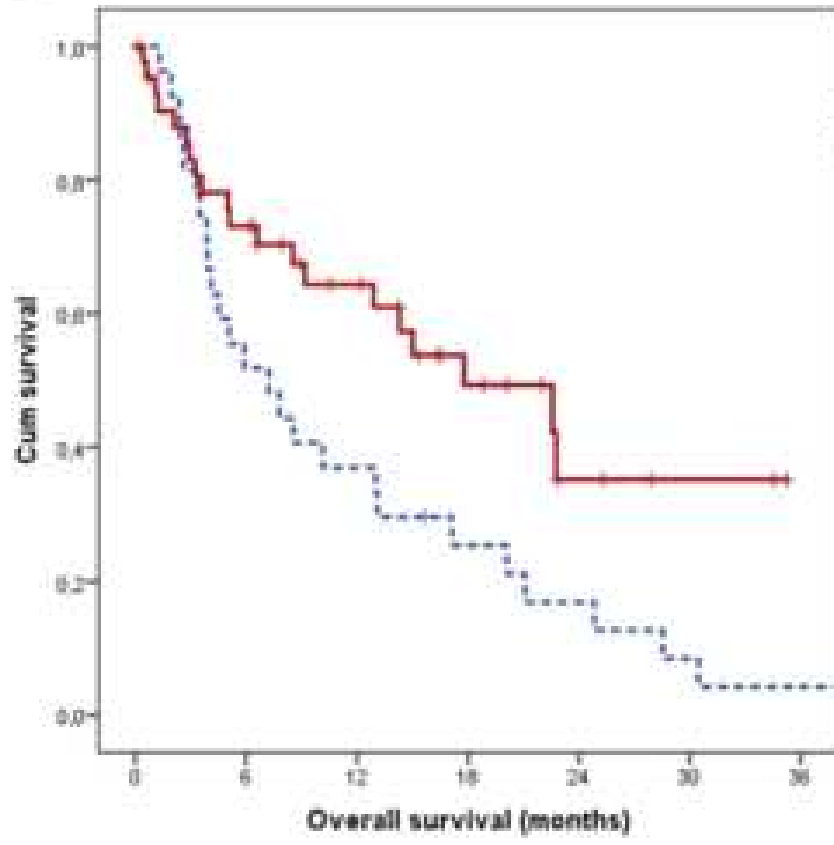

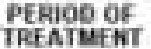

nowosion?

4 itos-36rz-censed

MEDIAN OS (months; $95 \%$ ca)

2008-2012:7.2(2.6-11.8)

2013-2017:17.7.(3.21.27.4)

Hit $0.485(0.271-0.882)$ p po.017 


\section{8 (Licensing of sorafenib in Italy)}

2008-2012 (timeline first half)

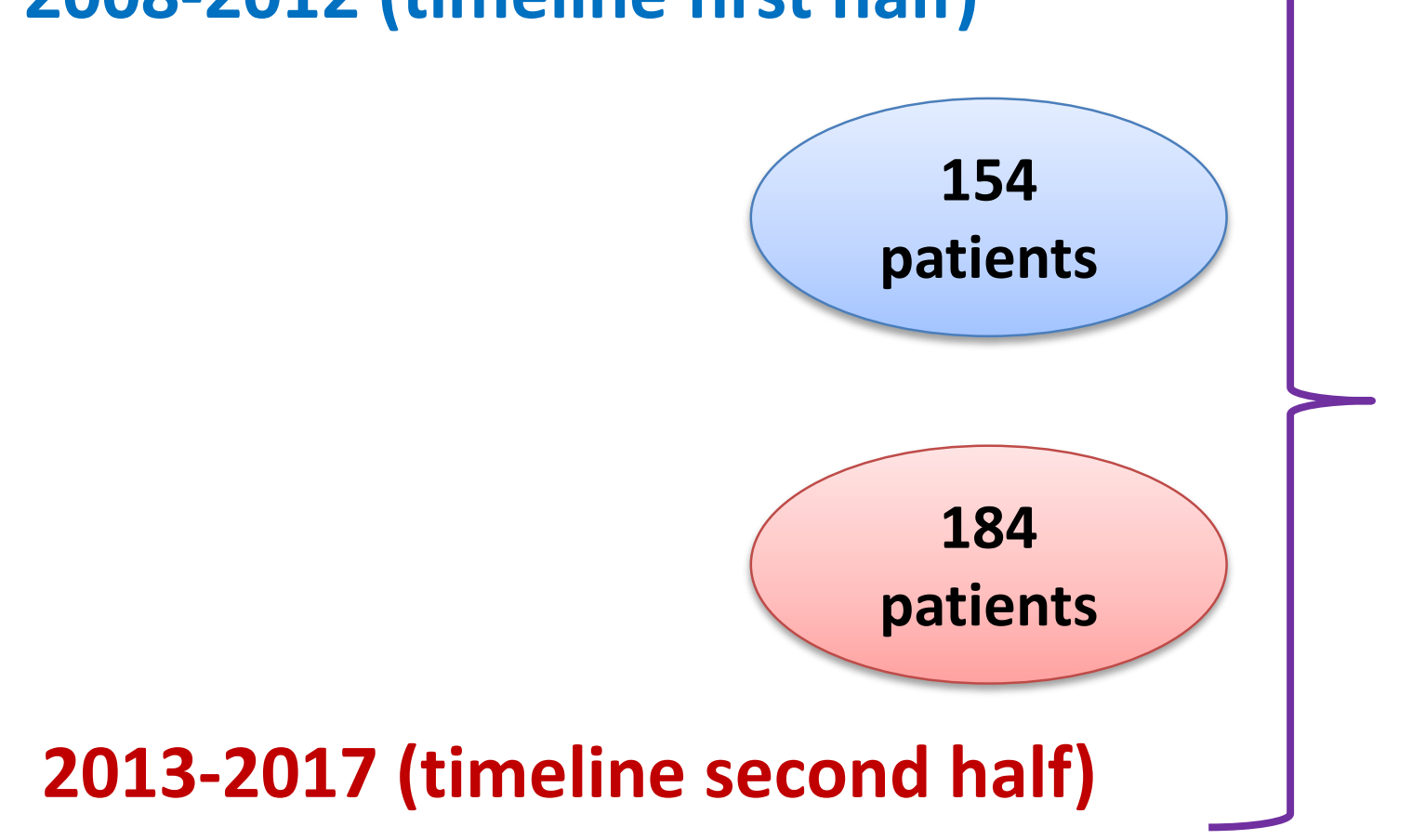

2017 (Licensing of regorafenib in Italy)

2013-2017 (timeline second half)
-Comparison of baseline characteristics -Comparison of follow up data -Censoring for second line treatments -Completeness of follow up analysis -Sensitivity analysis

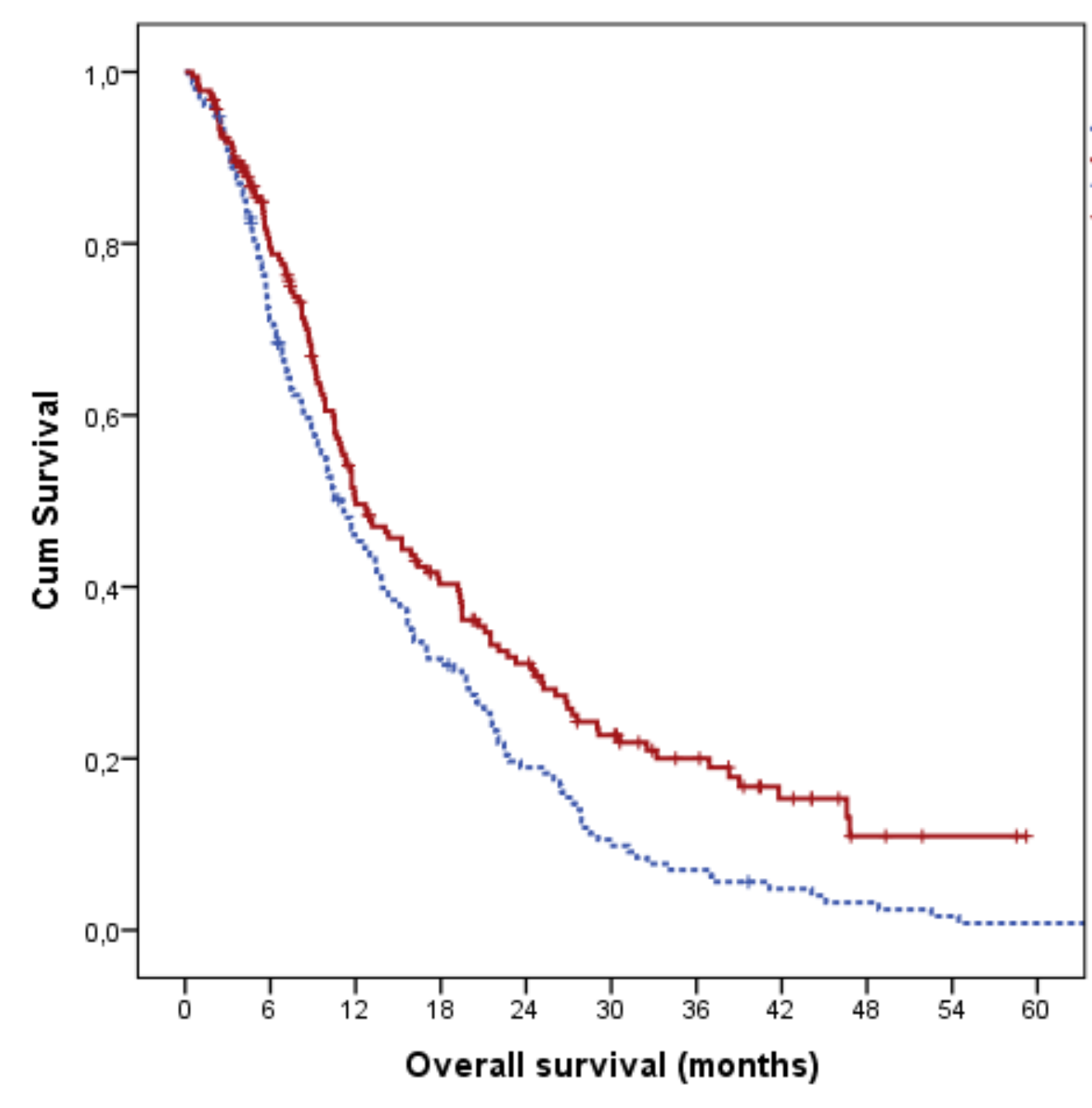

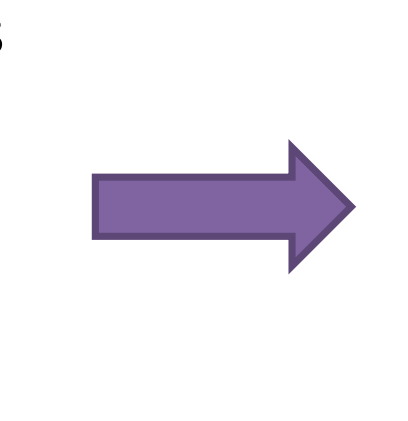


- Management of sorafenib-related adverse events has changed overtime

- A tailored approach with more temporary dose reductions is now more frequent

- Median treatment duration has increased overtime (5.8 vs 4.1 months)

- More importantly, overall survival has also increased (12.0 vs 11.0 months)

- Increasing survival impacts on the design of trials using sorafenib as comparator 

Click here to access/download
Supplementary material
SUPPLEMENTARY.docx Click here to access/download
Supplementary material
SUPPLEMENTARY.docx Click here to access/download
Supplementary material
SUPPLEMENTARY.docx

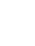

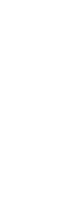 SUPPLEMENTARY.docX

\author{
SUPPLEMENTARY.docx
}


Click here to access/download

ICMJE disclosure form coi_CASADEI.pdf 
Click here to access/download

ICMJE disclosure form coi_granito.pdf 
Click here to access/download

ICMJE disclosure form coi_PISCAGLIA.pdf 
Click here to access/download

ICMJE disclosure form coi_tovoli.pdf 
Click here to access/download

ICMJE disclosure form coiFOSCHI.pdf 
Click here to access/download

ICMJE disclosure form coilELASI.pdf 
Click here to access/download

ICMJE disclosure form coiNEGRINI.pdf 
Click here to access/download

ICMJE disclosure form coiORSI.pdf 
Click here to access/download

ICMJE disclosure form coiRENZULLI.pdf 
Click here to access/download ICMJE disclosure form coiROVESTI.pdf 